\title{
Importance of cross-channel bathymetry and eddy viscosity parameterisation in modelling estuarine flow
}

\author{
Tjerk J. Zitman • Henk M. Schuttelaars
}

Received: 31 January 2011 / Accepted: 20 December 2011 /Published online: 11 February 2012

(C) The Author(s) 2012. This article is published with open access at Springerlink.com

\begin{abstract}
For a proper understanding of flow patterns in curved tidal channels, quantification of contributions from individual physical mechanisms is essential. We study quantitatively how such contributions are affected by crosschannel bathymetry and three alternative eddy viscosity parameterisations. Two models are presented for this purpose, both describing flow in curved but otherwise prismatic channels with an (almost) arbitrary transverse bathymetry. One is a numerical model based on the full threedimensional shallow water equations. Special feature of this diagnostic model is that assumptions regarding the relative importance of particular physical mechanisms can be incorporated in the computations by switching corresponding terms in the model equations on or off. We also present an idealized model that provides semi-analytical approximate solutions of the shallow water equations for all three considered alternative eddy viscosity parameterisations. It forms an aid in explaining and theorising about results obtained with the numerical model. Observations regarding Chesapeake Bay serve as a reference case for the present
\end{abstract}

Responsible Editor: Chari Pattiaratchi

This article is part of the Topical Collection on Physics of Estuaries and Coastal Seas 2010

\section{T. J. Zitman ( $\bowtie)$}

Department of Hydraulic Engineering,

Delft University of Technology,

Stevinweg 1,

2600 GA Delft, the Netherlands

e-mail: t.j.zitman@tudelft.nl

H. M. Schuttelaars

Delft Institute of Applied Mathematics,

Delft University of Technology,

Mekelweg 4,

2600 GA Delft, the Netherlands

e-mail: h.m.schuttelaars@tudelft.nl study. We find that the relative importance of both alongchannel advective forcing and transverse diffusive forcing depends on local characteristics of the cross-sectional bottom profile rather than global ones. In our reference case, tide-residual along-channel flow induced by these forcings is not small compared to the total tidal residual. Building on this observation, we present an indicative test to judge whether advective processes should be included in leading order in modelling tide-dominated estuarine flow. Furthermore, depending on the applied eddy viscosity parameterisation (uniformly or parabolically distributed over the vertical), we find qualitatively different spatial patterns for the along-channel advective forcing.

Keywords Tidal estuaries - Advective momentum transfer. Diagnostic modelling

\section{Introduction}

Various types of models have been developed to describe the flow in cross sections of tidal rivers and estuaries. Complex numerical models (e.g. Lerczak and Geyer 2004; Chen et al. 2009; Chen and Sanford 2009; Cheng et al. 2009) give detailed insight into the flow characteristics, but analysing the dominant physical processes is not straightforward. To acquire insight into the role of individual physical mechanisms, analytical models have been developed that focus on specific aspects of residual (e.g. Wong 1994; Valle-Levinson et al. 2003; Cheng and ValleLevinson 2009) or residual and tidal flow (e.g. Friedrichs and Hamrick 1996; Huijts et al. 2006, 2009, 2011) in estuarine cross sections. Recently, Burchard et al. (2011) adapted a numerical code that partly bridges the gap between complex numerical models on the one hand and analytical 
models on the other hand. The resulting numerical model includes many physical processes and state-of-the-art parameterisations. Although flow fields for individual driving mechanisms can be assessed separately, interpretation of physical mechanisms is still difficult.

Using highly idealized lateral bathymetries and eddy viscosity profiles, analytical models have been successful in explaining the physical mechanisms underlying many observed cross-sectional flow patterns. The insight gained with these idealized bottom profiles has been applied to more realistic cross-sectional bathymetries. Huijts et al. (2009) considered a cross section in the Chesapeake Bay and compared results obtained with an analytical model to observations gathered in this cross section. The order of magnitude of the tide-residual along-channel flow is reproduced correctly. An analysis based on velocity scales showed that the lateral density gradient plays a major role in the tide-residual cross-channel flow. For the alongchannel direction, they found advection to be one of the main mechanisms underlying the observed tide-residual flow. This advective contribution was obtained as a correction on the leading order tidal water motion, using an asymptotic expansion technique. The resulting crosssectional distribution of the tide-residual along-channel flow is more pronounced than that obtained from the observations, which show a seaward directed residual flow in a major part of the cross section. Near the bottom in both the centre of the channel and the inner bend, the observed residual flow is in the landward direction. In the model results, this landward residual flow is also present, but it is stronger than in the observations (around $0.15 \mathrm{~m} / \mathrm{s}$ in the model vs. $0.05 \mathrm{~m} / \mathrm{s}$ according to the observations) and it is spread over a larger area (Huijts et al. 2009, Fig. 2). This may indicate that the applied first-order correction somewhat exaggerates the advective forcing, resulting in an overestimation of the landward residual flow.

For a laterally idealized geometry, the analytic model results of Huijts et al. (2009) are reproduced using a fully non-linear model by Schramkowski et al. (2007) under specific conditions. For a sufficiently large tidal discharge, Schramkowski et al. (2007) find that in computing residual flow, advection cannot be neglected in leading order. In low flow conditions, on the other hand, advective forcing appears to be of relatively low importance. This seems to disagree with the results obtained by Huijts et al. (2009) for a realistic cross-sectional bathymetry. For comparatively weak tidal conditions, they found advection to play a substantial role in the residual flow pattern. The difference in cross-channel bottom topography may be part of an explanation for this discrepancy. The model computations of Schramkowski et al. (2007) concern a smooth, mildly sloping bottom, whereas Huijts et al. (2009) considered a channel with a somewhat craggy bottom where gentle slopes are alternated by steep parts (their Fig. 2). This suggests that the importance of advection might increase with the steepness of the transverse bottom profile.

To investigate the influence of transverse topography on flow patterns in the cross section, with an emphasis on advective momentum transfer, we develop two diagnostic models. One of them is more advanced than most analytical models as it solves the fully non-linear shallow water equations. It is henceforth referred to as advanced model (AM). Following Burchard et al. (2011), a special feature of this model is that individual terms in the basic model equations, each representing a specific physical mechanism, can be switched on or off. This facilitates quantification of the contributions of these mechanisms to the flow pattern. Other than the model of Burchard et al. (2011), however, our model includes Coriolis forcing, river discharge and effects of curvature of the channel.

The other diagnostic model is based on a simplification of the shallow water equations. It is referred to as idealized model (IM), and it is meant to facilitate understanding and explaining features encountered in observed and computed flow patterns. This model concerns a straight, prismatic channel, and advective processes are assumed negligible compared to local accelerations and internal friction.

Apart from idealizing the cross-sectional bathymetry, most idealized models use an eddy viscosity that is time-invariant and constant over the water column. Burchard et al. (2011), on the other hand, deploy a state-of-the-art formulation for the vertical eddy viscosity in an estuarine cross section with highly idealized geometry. In that formulation, eddy viscosity is time-dependent and non-uniform in the vertical. To assess the importance of eddy viscosity formulation on computed flow fields, we focus, as a first step, on a time-invariant but vertically varying distribution. This implies that circulations due to tidal straining are not considered. Although the vertical distribution of eddy viscosity can take any prescribed shape, we focus on only three alternatives. One of them is a parabolic distribution over the entire water column. Another is a vertically invariant eddy viscosity. The third alternative is a combination of a parabolic distribution in a small region just above the bottom and vertically invariant further up in the water column. This latter shape is used to simulate a partial-slip bottom boundary condition in the AM (Schramkowski et al. 2010). In all three alternatives, the eddy viscosity scale (its depth-averaged value) may vary in transverse direction. However, both shape and scale are taken time-invariant. Roughly, these three alternatives cover the most frequently used parameterisations of the eddy viscosity profile (also see Bowden 1965). By comparing results obtained with these parameterisations, we can investigate the sensitivity of computed flow patterns to the choice of turbulence closure.

As we intend to compare our results with those obtained with an analytical model and observations, we focus on a 
tidal channel that has been studied previously with the aid of such a model. In this respect, we have chosen to build on the work of Huijts et al. (2009) concerning Chesapeake Bay. They evaluate and analyse their model results against flow velocities and density gradients observed and described extensively by Fugate et al. (2007). For the present study, we use the same data as a reference.

The joint deployment of an advanced and an idealized model is to facilitate identification and understanding of the relative importance of specific individual physical mechanisms in the conditions investigated as well as to indicate the implications of these findings in a broader perspective. More particularly and summarising the above, the aim of our study is twofold. It is:

- To assess the influence of cross-channel topography on the along-channel flow structure, with an emphasis on advective momentum transfer

- To depict the influence of eddy viscosity parameterisation in this respect

\section{Advanced model}

The model used for the present investigation simulates the flow in a cross section of a (tidal) channel. The geometry of the cross section consists of a schematised bathymetry, comprising a smoothly varying bottom with vertical walls on either side of the channel so that the bottom is never uncovered. Although parts of the bottom may be steep, vertical jumps cannot be accommodated. Apart from this restriction, the bottom profile can attain an arbitrary shape. The geometry of the cross section as well as the flow itself and the prescribed density gradients are assumed uniform in along-channel direction. In the horizontal plane, the channel may be either straight or circular (see Fig. 1 for an example).

In our model, the flow is forced by a combination of prescribed transverse and longitudinal density gradients and a prescribed longitudinal pressure gradient due to a surface

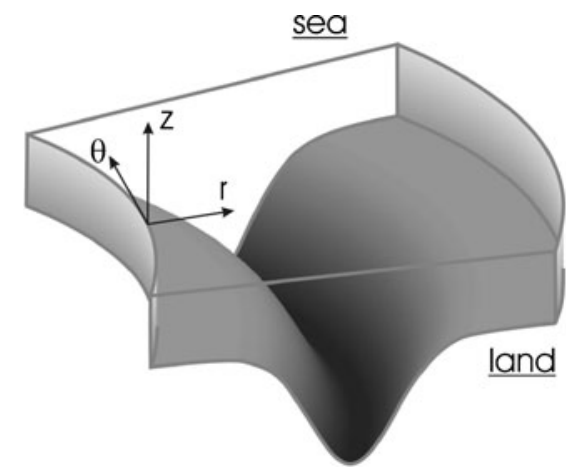

Fig. 1 Schematised geometry of a tidal channel. The $(r, \theta, z)$ coordinate system is used in the AM with $\theta$ pointing seaward slope. These quantities are obtained from observations. Wind shear stress is not included. Instead of a longitudinal surface slope, a total discharge may be prescribed. In that case, this surface slope is chosen such that the prescribed discharge is produced. Furthermore, the rigid-lid approximation is applied, meaning that spatial and temporal variations of the surface level elevation are neglected, although pressure gradients due to such variations are taken into account.

The flow is described with the three-dimensional shallow water equations. For this purpose, we use a coordinate system as indicated in Fig. 1, with polar coordinates in the horizontal plane. In all model results discussed in the present paper, the $\theta$ coordinate increases towards the seaward end of the tidal channel. Including the assumption of alongchannel uniformity and the rigid-lid approximation, the model equations read

$$
\begin{aligned}
\frac{\partial u_{r}}{\partial t} & +u_{r} \frac{\partial u_{r}}{\partial r}+u_{z} \frac{\partial u_{r}}{\partial z}-\gamma u_{\theta}-\frac{u_{\theta}^{2}}{r}= \\
& +z \frac{g}{\rho_{0}} \frac{\partial \rho}{\partial r}-g \frac{\partial \zeta}{\partial r}+v_{\mathrm{t}} \frac{\partial}{\partial z}\left(\varphi_{z} \frac{\partial u_{r}}{\partial z}\right)+v_{\mathrm{h}} \frac{1}{r} \frac{\partial}{\partial r}\left(r \frac{\partial u_{r}}{\partial r}\right)
\end{aligned}
$$

$$
\begin{aligned}
\frac{\partial u_{\theta}}{\partial t} & +u_{r} \frac{\partial u_{\theta}}{\partial r}+u_{z} \frac{\partial u_{\theta}}{\partial z}+\gamma u_{r}+\frac{u_{r} u_{\theta}}{r}= \\
& +\frac{z}{r} \frac{g}{\rho_{0}} \frac{\partial \rho}{\partial \theta}-g \frac{1}{r} \frac{\partial \zeta}{\partial \theta}+v_{\mathrm{t}} \frac{\partial}{\partial z}\left(\varphi_{z} \frac{\partial u_{\theta}}{\partial z}\right)+v_{\mathrm{h}} \frac{1}{r} \frac{\partial}{\partial r}\left(r \frac{\partial u_{\theta}}{\partial r}\right)
\end{aligned}
$$

and

$$
\frac{\partial u_{r}}{\partial r}+\frac{\partial u_{z}}{\partial z}=0
$$

reflecting momentum conservation in $r$ and $\theta$ direction and continuity, respectively. In the above expressions; $r, \theta$ are cross-channel and along-channel coordinates; $z$ is a vertical coordinate ( $z=0$ at the free surface); $t$ is time; $u_{r}, u_{\theta}, u_{z}$ are cross-channel, along-channel and vertical flow velocity components; $\zeta$ is surface level elevation; $\rho$ is mass density of the fluid $\left(\rho_{0}\right.$ is a reference density, set at $\left.1,000 \mathrm{~kg} / \mathrm{m}^{3}\right) ; \gamma$ is the Coriolis parameter; $v_{\mathrm{t}}$ is depth-mean vertical eddy viscosity; $\varphi_{z}$ is shape function for the vertical distribution of eddy viscosity (normalized such that its depth-mean value equals 1$) ; v_{\mathrm{h}}$ is horizontal eddy viscosity (time- and space-invariant) and $g$ is gravitational acceleration.

Corresponding boundary conditions comprise zero $u_{r}$ and $u_{\theta}$ at the two sidewalls. Along the bottom, a no-slip condition is applied to the tangential velocity component, and as no water can pass into or out of the bottom, a zero normal velocity component is imposed. Put together this means that 
at the bottom, all three velocity components are set to zero. As wind shear stress is not included in the model, vertical gradients of the two horizontal velocity components at the water surface are set to zero. Furthermore, a kinematic boundary condition is imposed at the surface. As we apply the rigid-lid approximation, this boils down to demanding a zero vertical velocity at the surface.

Another consequence of the rigid-lid approximation is that the depth-integrated transverse velocity equals zero at every location in the cross section. This follows from integrating the continuity Eq. 3 over the water depth:

$$
\int_{-h}^{0} \frac{\partial u_{r}}{\partial r}+\frac{\partial u_{z}}{\partial z} \mathrm{~d} z=\frac{\partial}{\partial r} \int_{-h}^{0} u_{r} \mathrm{~d} z-u_{r}(-h) \frac{\partial h}{\partial r}+u_{z}(0)-u_{z}(-h)=0
$$

in which $z=-h$ refers to the bottom. From the imposed boundary conditions, it follows that $u_{r}(-h)=u_{z}(-h)=u_{z}(0)=$ 0 . Substituting this into the above expression yields

$$
\frac{\partial}{\partial r} \int_{-h}^{0} u_{r} \mathrm{~d} z=0
$$

Combining this with the boundary condition that $u_{r}=0$ at both side walls of the channel shows that in the present approach application of the rigid-lid approximation implies a zero net transverse flow at every location along the transect. We use this property to have our model resolve the time- and space-dependent cross-channel pressure gradient.

Flow velocity components and the transverse pressure gradient are solved numerically using a collocation method based on Chebyshev polynomials (see, e.g. Boyd 2000). In the physical domain, we use a non-orthogonal curvilinear grid. For integration in the time domain, a fully implicit finite difference approximation is applied. As the model equations are non-linear in the flow velocity components, a Newton-Raphson iteration is performed in each time step (see, e.g. Abramowitz and Stegun (1970); application is depicted in "Appendix 1").

As we intend to compare our model results with the findings of Huijts et al. (2009), we mention three noticeable differences between their model and ours:

- Our model does not include wind forcing.

- Advection is taken into account in leading order in our model, whereas Huijts et al. (2009) include it in first order.

- Boundary layers near the side walls of the channel are resolved by our model as we include horizontal diffusion and impose a zero transverse velocity at these walls. These boundary layers are not included in the model presented by Huijts et al. (2009).
Where appropriate, these differences are considered in the intended comparison.

\section{Idealized model}

As an aid to better understand the role of individual flowdriving mechanisms, we introduce an idealized analytical model (IM). Compared to the AM, the idealization rests in the assumption that both advection and transverse diffusion can be neglected in leading order. Furthermore, we focus on a straight channel. This way, the basic equations of the IM read

$$
\begin{aligned}
\frac{\partial u_{x}}{\partial t}+\gamma u_{y} & =-(\zeta-z) \frac{g}{\rho_{0}} \frac{\partial \rho}{\partial x}-g \frac{\partial \zeta}{\partial x}+\beta \frac{\partial}{\partial \sigma}\left(\varphi \frac{\partial u_{x}}{\partial \sigma}\right) ; \\
u_{x}\left(\sigma_{0}\right) & =\left.\frac{\partial u_{x}}{\partial \sigma}\right|_{\sigma=1}=0 \\
\frac{\partial u_{y}}{\partial t}-\gamma u_{x} & =-(\zeta-z) \frac{g}{\rho_{0}} \frac{\partial \rho}{\partial y}-g \frac{\partial \zeta}{\partial y}+\beta \frac{\partial}{\partial \sigma}\left(\varphi \frac{\partial u_{y}}{\partial \sigma}\right) ; \\
u_{y}\left(\sigma_{0}\right) & =\left.\frac{\partial u_{y}}{\partial \sigma}\right|_{\sigma=1}=0
\end{aligned}
$$

in which the $x$ is the along-channel coordinate and $y$ is the transverse coordinate. The vertical coordinate $\sigma$ is scaled such that $\sigma=0$ at the bottom and $\sigma=1$ at the surface. A noslip bottom boundary condition is applied (at $\sigma=\sigma_{0}$ ) and as we disregard effects of wind, the vertical gradient of the horizontal flow velocity components at the surface is set to zero.

The shape of the vertical eddy viscosity profile is given by $\varphi$ (which is the equivalent of $\varphi_{z}$ for the scaled vertical coordinate $\sigma$ ). The corresponding scale factor $v_{\mathrm{t}}$ (see Eqs. 1 and 2) is incorporated in the parameter $\beta$, defined as

$\beta=\frac{h^{2}}{\bar{v}_{\mathrm{t}}}$

Like in the AM, we apply the rigid-lid approximation. As explained in the section on the AM, this boils down to demanding a zero net transverse flow:

$$
\int_{\sigma_{0}}^{1} u_{y} \mathrm{~d} z=0
$$

Semi-analytical solutions to Eqs. 6, 7, 8 and 9 for both steady and harmonically varying density gradients and along-channel surface slopes are given in "Appendix 3" and "Appendix 4". An essential approximation in the derivation of these solutions is that the shape $\varphi$ of the vertical distribution of eddy viscosity is invariant in time.

Equations 6, 7, 8 and 9 apply to a single point in the horizontal plane. Repeated application of these equations for 
a series of points along a transect of a channel yields an image of the flow pattern in that transect. Importantly, however, this pattern includes the assumption that transverse advection can be neglected in leading order.

We use this image to compute a first-order estimate of cross-channel advective forcing. Flow profiles obtained for two consecutive points along the transect are used to estimate transverse derivatives of the horizontal velocity components. The so-obtained $\partial u_{y} / \partial y$ is substituted into the continuity equation to arrive at an estimate of the vertical velocity component $u_{z}$. Including a no-slip bottom boundary condition, the result reads

$u_{z}(s)=-\int_{z_{\mathrm{b}}}^{s} \frac{\partial u_{y}}{\partial y} \mathrm{~d} z$

in which $z_{\mathrm{b}}$ is the bottom level elevation. Subsequently, a first-order estimate of along-channel advective forcing is obtained from

$u_{y} \frac{\partial u_{x}}{\partial y}+u_{z} \frac{\partial u_{x}}{\partial z}$

This procedure is elaborated in detail in "Appendix 3" for flow driven by both steady and harmonically varying along and cross-channel density gradients and along-channel surface slope. In a similar fashion, a first-order estimate of transverse diffusion of the along-channel velocity component $\left(v_{\mathrm{h}} \partial^{2} u_{x} / \partial y^{2}\right)$ is determined (see "Appendix 4").

A so-obtained first-order estimate of along-channel advective forcing can be used to correct computed flow velocities for effects of advection. Huijts et al. (2009) have performed such a correction. In the present study, we focus on the spatial pattern of along-channel advection and how that is influenced by eddy viscosity parameterisation. In addition, we address the possibility of an a priori evaluation of the relative importance of advection based on the firstorder estimate (see under Section 6 in this paper).

\section{Turbulence closure}

In the basic model Eqs. 1 and 2 for the AM and Eqs. 6 and 7 for the IM, we have written the vertical distribution of eddy viscosity as the product of a depth-mean value $v_{\mathrm{t}}$ and a shape function $\varphi$. In the present paper, we focus on three options for this shape function. One of them concerns a parabolic variation of the eddy viscosity over the vertical, and in another option, the eddy viscosity is taken vertically invariant (Bowden 1965). The latter is included for compatibility with the idealized model of Huijts et al. (2009). The two options are depicted in Fig. 2 a, c, respectively.
The profile given in Fig. 2 b is used in the present study to simulate a partial-slip bottom boundary condition. These three options are discussed briefly in "Appendix 6".

The parabolic eddy viscosity distribution serves as a reference for all model computations discussed in the present paper. To evaluate the consequences of choosing a different type of eddy viscosity parameterisation, it is necessary to formulate a criterion to arrive at mutually comparable depth-mean values for the eddy viscosity. In the literature, various options for such a criterion are encountered. For the present investigation, we have chosen to demand that the various parameterisations must yield identical energy dissipation rates, as mentioned for instance by Ianniello (1979). To arrive at an explicit relation between the eddy viscosity scales, we apply this demand to plane shear flow (see Eqs. 98 and 99). The corresponding dissipation rate $P$ per unit of horizontal area reads

$$
P=\int_{\sigma_{0}}^{1} u \frac{\mathrm{d} \tau}{\mathrm{d} \sigma} \mathrm{d} \sigma \quad ; \quad \tau=\rho \bar{v}_{t} \varphi \frac{1}{h} \frac{\mathrm{d} u}{\mathrm{~d} \sigma}
$$

in which $\tau$ is the shear stress. Evaluating Eq. 12 for both considered types of eddy viscosity distribution and subsequently demanding equality with respect to the resulting dissipation rates $P$ yields the required relation between the eddy viscosity scales corresponding to both distributions.

\section{Model results}

Our investigation starts with fitting our AM to the field data concerning Chesapeake Bay (Fugate et al. 2007), assuming that eddy viscosity is distributed parabolically over the vertical. In the model computations, we have used parameter values as listed in Table 1. Most of these values are identical to those mentioned by Huijts et al. (2009). Wind forcing, however, is neglected. On the other hand, following the recommendation of Huijts et al. (2011), we include an $\mathrm{M}_{2}$ variation of the cross-channel density gradient. It is uniform in lateral direction, and its amplitude is chosen equal to the cross-sectional average derived from the observations $\left(\sim 10^{-3} \mathrm{~kg} / \mathrm{m}^{4}\right)$.

The bottom roughness is assumed time- and spaceinvariant. It is represented by a roughness length (denoted by $k_{\mathrm{s}}$; see Eq. 101 in "Appendix 6"). It is used to determine the relative height $\sigma_{0}$ above the bottom at which the bottom boundary conditions are to be applied in conformity with the turbulence closure scheme (see Section 4). The value of $k_{\mathrm{s}}$ is typically in the order of a few $\mathrm{cm}$, leading to a $\sigma_{0}$ of around 0.001 for the Chesapeake Bay case. For the present study, we have assumed that $k_{\mathrm{s}}=0.05 \mathrm{~m}$. A sensitivity test based on the IM has indicated that model results show negligible variation for $\sigma_{0}$ in the range of 0.0005 to $0.05 \mathrm{~m}$. 
Fig. 2 Three options for a prescribed vertical distribution of eddy viscosity: a parabolic shape $(a)$, a combined parabolic and vertically invariant profile (b) and a uniform distribution (c). All three options are applied in the AM in combination with a no-slip bottom boundary condition. Option $(b)$ is meant to simulate partial slip at $\sigma=\sigma_{\mathrm{b}}$. Profiles $a$ and $c$ are used in the IM

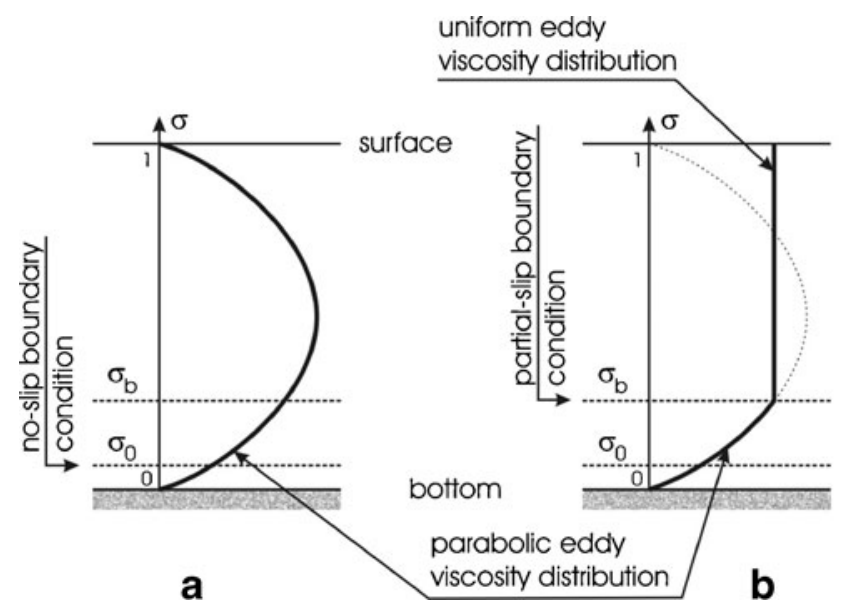

vertically invariant eddy viscosity

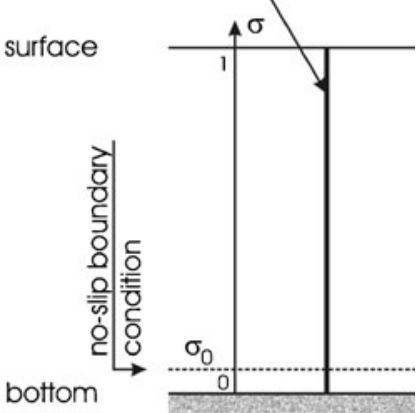

C
On average, the observations exhibit a tide-residual seaward flow. This is caused by river discharge. However, in the centre of the channel, a landward flow occurs in the lower part of the water column. This area of landward flow extends along the bottom of the channel towards the side at the inner bend (see Fig. 4a). The main features of this flow pattern can be explained by considering gravitational circulation and river discharge. This combination involves two flow-driving forces. One of them is related to an alongchannel density gradient. It is zero at the surface and increases with depth. The other force comes from an along-channel surface slope and is depth-invariant. These two forces and their attended flows are in opposite direction. In Chesapeake Bay, the density gradient acts landward, and the surface slope is directed seaward. Their cross-sectional integrated effect yields the river discharge.

Due to the difference in vertical distribution of the two forces, a density gradient-dominated flow is encountered in the lower part of the water column, whereas the opposing flow in the upper part is dominated by the surface slope. Vertical exchange of momentum has a smoothening effect on this pattern. It reduces spatial differences in alongchannel flow velocities without affecting the net result (i.c. the river discharge). An increase of this momentum exchange yields an overall reduction of flow velocities. As a result, a larger part of the channels cross section is needed to accommodate the river discharge. The density gradientdominated flow is confined to the remaining part of that cross section. This part tends to concentrate around the deeper, central part of the cross section as only in the lower part of the water column the flow-driving force related to the density gradient dominates over that resulting from the surface slope. This mechanism is addressed in detail in "Appendix 5" with illustrative applications of the idealized model IM.

The above reasoning forms the basis for calibrating our AM to the observed tide-residual along-channel flow presented in Fig. 4. In all our model computations, the alongchannel density gradient and river discharge are prescribed based on observations. Vertical exchange of momentum is reflected by eddy viscosity. In tuning our model, we assume a parabolic distribution of eddy viscosity over the vertical

Table 1 Parameter values used in model computations

\begin{tabular}{|c|c|c|c|c|c|}
\hline \multirow[t]{2}{*}{ Parameter } & \multirow[t]{2}{*}{ Unit } & \multirow[t]{2}{*}{ Value } & \multirow[t]{2}{*}{$M_{0}$ component } & \multicolumn{2}{|c|}{$M_{2}$ component } \\
\hline & & & & Amplitude & Phase (rad) \\
\hline Along-channel discharge ${ }^{\mathrm{a}}$ & $\mathrm{m}^{3} / \mathrm{s}$ & & 900 & 9,500 & 0 \\
\hline Along-channel density gradient ${ }^{\mathrm{a}}$ & $\mathrm{kg} / \mathrm{m}^{4}$ & & $4 \times 10^{-4}$ & - & - \\
\hline Cross-channel density gradient ${ }^{\mathrm{a}}$ & $\mathrm{kg} / \mathrm{m}^{4}$ & & $-0.5 \times 10^{-4} \ldots-5 \times 10^{-4}$ (the transverse profile is given in Fig. 3 ) & $10^{-3}$ & $\pi / 2$ \\
\hline Gravitational acceleration $^{\mathrm{b}}$ & $\mathrm{N} / \mathrm{kg}$ & 9.81 & & & \\
\hline Coriolis parameter ${ }^{\mathrm{b}}$ & $\mathrm{s}^{-1}$ & $10^{-4}$ & & & \\
\hline Horizontal diffusion coefficient ${ }^{\mathrm{b}}$ & $\mathrm{m}^{2} / \mathrm{s}$ & 1 & & & \\
\hline Bottom roughness length $^{\mathrm{b}}$ & $\mathrm{m}$ & 0.05 & & & \\
\hline Period of semi-diurnal tide ${ }^{b}$ & s & 44,712 & & & \\
\hline Radius of curvature $^{\mathrm{b}}$ & $\mathrm{km}$ & 40 & & & \\
\hline
\end{tabular}

${ }^{a}$ The quantities listed are related to prescribed flow-driving forces

${ }^{\mathrm{b}}$ Other parameters 


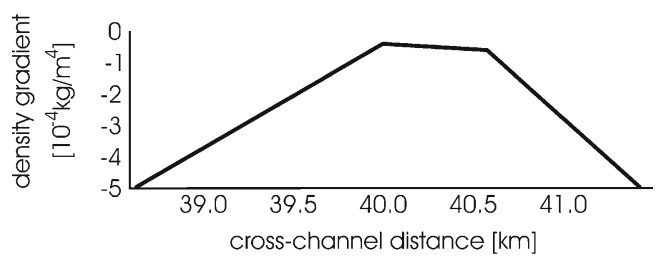

Fig. 3 Cross-channel gradient of the depth-mean density as used in all model computations discussed in the present paper. The density increases towards the outer bend. Its gradient varies between a minimum of $0.5 \times 10^{-4} \mathrm{~kg} / \mathrm{m}^{4}$ and a maximum of $5.0 \times 10^{-4} \mathrm{~kg} / \mathrm{m}^{4}$. This transverse profile is a schematisation, based on the observations by Fugate et al. (2007)

and choose the corresponding scale such that the computed tide-residual along-channel flow agrees well with the observations (Fig. 4a). Model results obtained for eddy viscosity scales of $0.002,0.006$ and $0.010 \mathrm{~m}^{2} / \mathrm{s}$ are given in Fig. 5 . Of these three values, $0.006 \mathrm{~m}^{2} / \mathrm{s}$ yields the best agreement with respect to order of magnitude and direction of the tideresidual along-channel flow velocity. In computations where we assume a uniform distribution of eddy viscosity, we use a scale of $0.0015 \mathrm{~m}^{2} / \mathrm{s}$. With these scales, both distributions yield the same dissipation rate (see Section 4).

As we use the observations as a reference for our investigating concerning the relative importance of advection and transverse bottom slope, a more subtle tuning of the model to the observations than described above has not been carried out. In Fig. 6, computed $M_{0}$ and $M_{2}$ components of the along and cross-channel velocity components for the run with a depth-averaged eddy viscosity of $0.006 \mathrm{~m}^{2} / \mathrm{s}$ are given. A comparison with observational results (Fig. 4) shows that orders of magnitude are well reproduced by the model, as well as the overall spatial pattern. A poor agreement is encountered for the $M_{0}$ velocity components in the area near the inner bend of the channel (left-hand side of Fig. 4a, b).

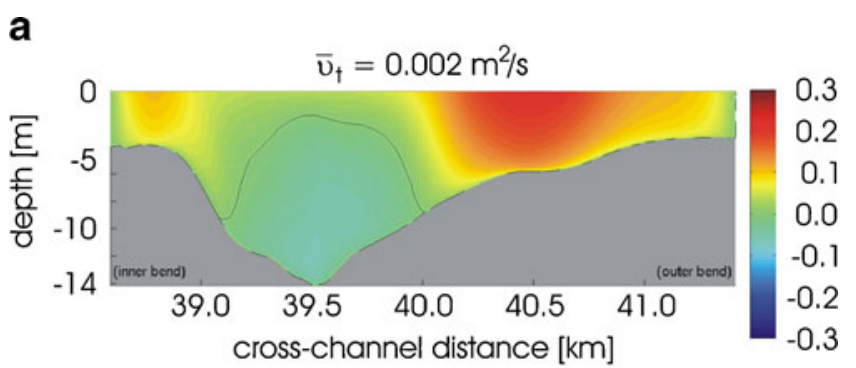

b
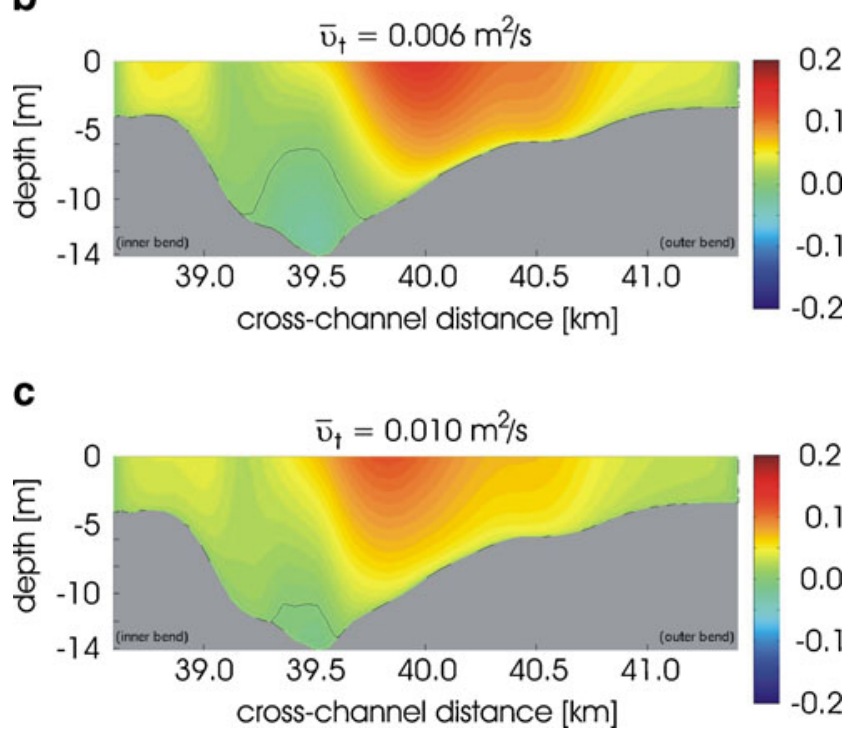

Fig. 5 Tide-residual along-channel flow computed in three calibration runs. A parabolic eddy viscosity distribution is assumed. a-c A depthmean eddy viscosity of $0.002,0.006$ and $0.010 \mathrm{~m} 2 / \mathrm{s}$, respectively. Black curves are zero velocity contours. Colour scales are in metres per second and warm colours indicate a seaward flow

In this area, the observed residual landward flow near the bottom of the channel is not reproduced correctly. That holds also for the tide-residual transverse flow in this area
Fig. $4 M_{0}$ (a, b; warm colours indicate a seaward flow for the along-channel component or to the right for the cross-channel component) and $M_{2}$ (c, d) amplitudes of the observed along-channel $(\mathbf{a}, \mathbf{c})$ and crosschannel $(\mathbf{b}, \mathbf{d})$ velocity components. Black curves are zero velocity contours
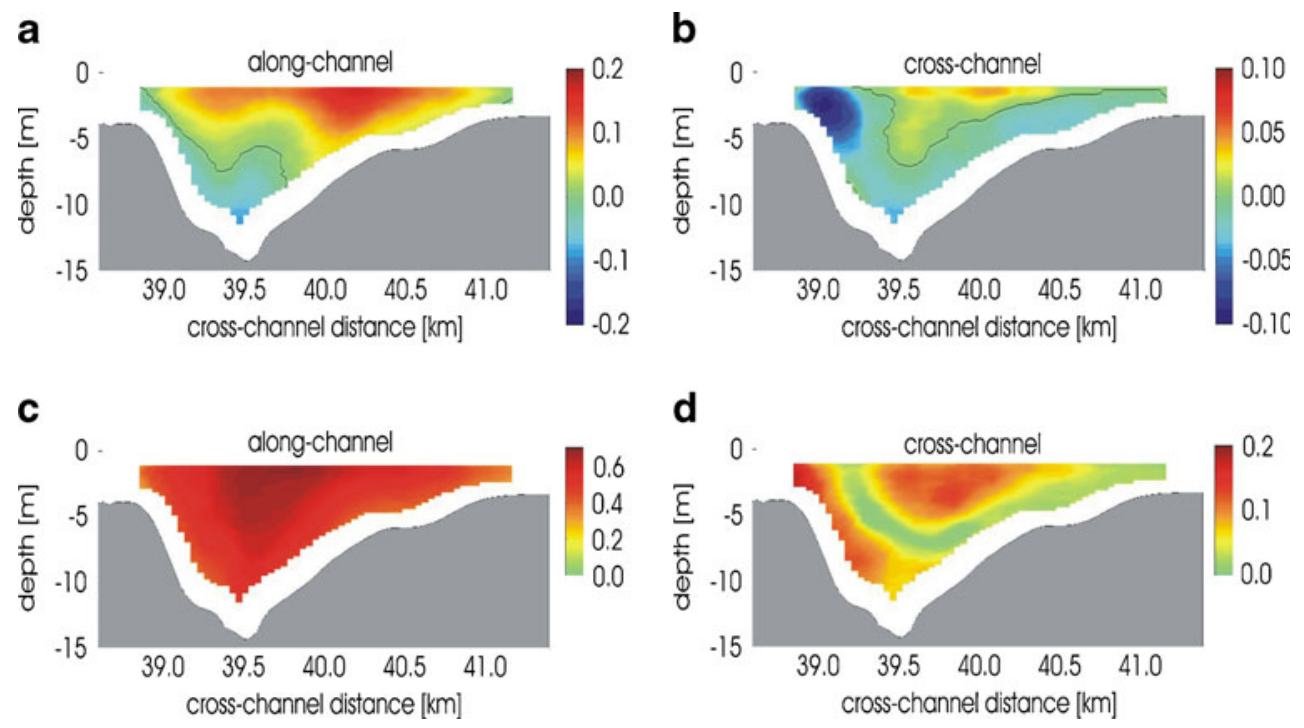
Fig. $6 M_{0}(\mathbf{a}, \mathbf{b}$; warm colours indicate a seaward flow for the along-channel component or to the right for the cross-channel component, and black curves are zero velocity contours) and amplitudes of $M_{2}(\mathbf{c}, \mathbf{d})$ components of the alongchannel (a, c) and cross-channel (b, d) velocity (in metres per second) computed assuming a parabolic eddy viscosity distribution and corresponding depth-mean value of $0.006 \mathrm{~m}^{2} / \mathrm{s}$
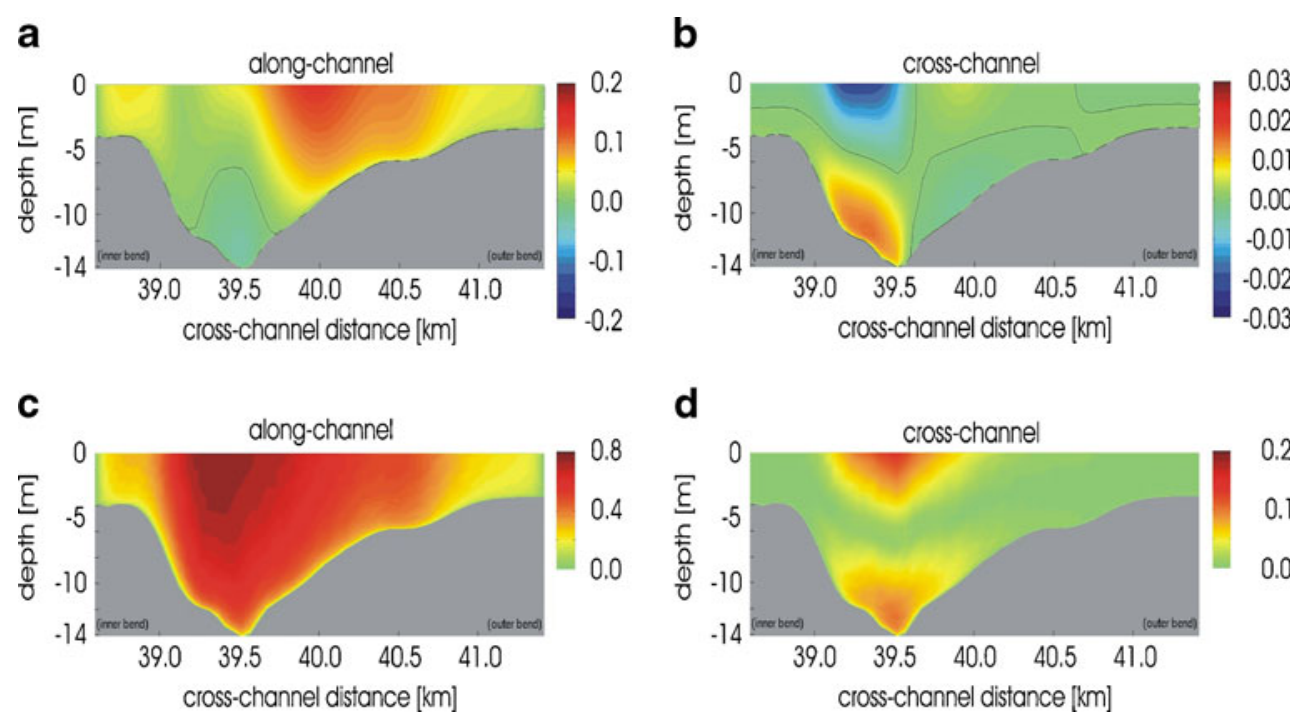

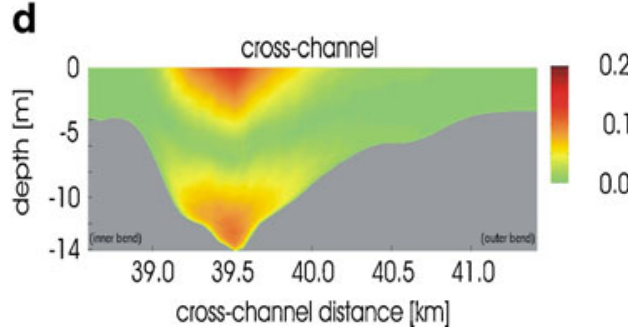

(see Fig. 6b). Observations near the inner bend show a flow towards the centre of curvature of the channel (to the left in the figure) that occurs in the entire water column. A possible explanation is that the rigid-lid approximation is inadequate in this comparatively shallow area, as a result of which the assumption of along-channel uniformity does not apply. Simultaneously, the lateral flow towards this area that occurs when during flood the water level rises is attended with a smaller water depth than the return flow during ebb. As a result, the flood flow is stronger than the ebb flow. The net effect shows as a residual in up slope direction. It is inherently impossible for our model to reproduce this phenomenon. As we apply the rigid-lid approximation, the depthaveraged cross-channel velocity equals zero at all times. This means that near the side walls, there is either no flow or a vertical circulation. A flow towards the side during one part of the tide and away from that wall in another part is not possible in our model concept. An alternative possibility is that a vertical circulation actually occurs in reality, but that its near-bottom down slope branch does not show in the observations as it happens to be outside the observational domain (the blank areas in Fig. 4).

Similar considerations apply to the discrepancy between observed and computed $M_{2}$ components of the crosschannel velocity (Figs. 4d and 6d, respectively). Apart from these qualitative considerations, no attention has been paid in the present study to these local discrepancies between observations and model output.

In our study on the role of eddy viscosity parameterisation, we compare the results of three computations with the AM:

1. A parabolic eddy viscosity distribution $\left(\bar{v}_{\mathrm{t}}=0.006 \mathrm{~m}^{2} / \mathrm{s}\right)$ and including advection (this is the result of tuning the AM)

2. A uniform eddy viscosity distribution of $\bar{v}_{\mathrm{t}}=0.0015 \mathrm{~m}^{2} / \mathrm{s}$. According to Eq. 12, this yields similar dissipation as the combination of a parabolic distribution with a scale of $0.006 \mathrm{~m}^{2} / \mathrm{s}$

3. A combined parabolic and vertically invariant eddy viscosity (see Fig. 2 b) to simulate a partial-slip bottom boundary condition. The eddy viscosity scale is set at $\bar{v}_{\mathrm{t}}=0.0015 \mathrm{~m}^{2} / \mathrm{s}$

We use the model results for these three cases also to assess the relative importance of advection. For this purpose, we decompose computed tide-residual horizontal flow velocity components into contributions that can be ascribed to individual flow-driving mechanisms. We discriminate between flows induced by advection, Coriolis effect, density gradients, along-channel net discharge (i.c. river discharge and Stoke's drift together), curvature and lateral diffusion. The decomposition method is explained in "Appendix 2".

Tide-residual along and cross-channel flow velocities and corresponding decompositions are presented in Figs. 7 and 8 for model computations using a parabolic and for a uniform eddy viscosity distribution, respectively. Figure 9 shows the tide-residual advective forcing computed from model results concerning both viscosity parameterisations. Results obtained for the simulation of a partial-slip bottom boundary condition are not shown in the present paper. These results are much alike those found for a uniform eddy viscosity distribution. The differences are not essential in view of our present research aims.

From Figs. 7 and 8, it can be seen that assuming a uniform eddy viscosity distribution yields larger tideresidual flow velocities than when a parabolic profile is assumed, but the spatial patterns are much alike. The difference may be explained partly by the applied eddy viscosity scales. We have related these scales to one another such that they yield identical dissipation rates in the case of steady, plain shear flow. This may be insufficient for the complex flow at hand. 


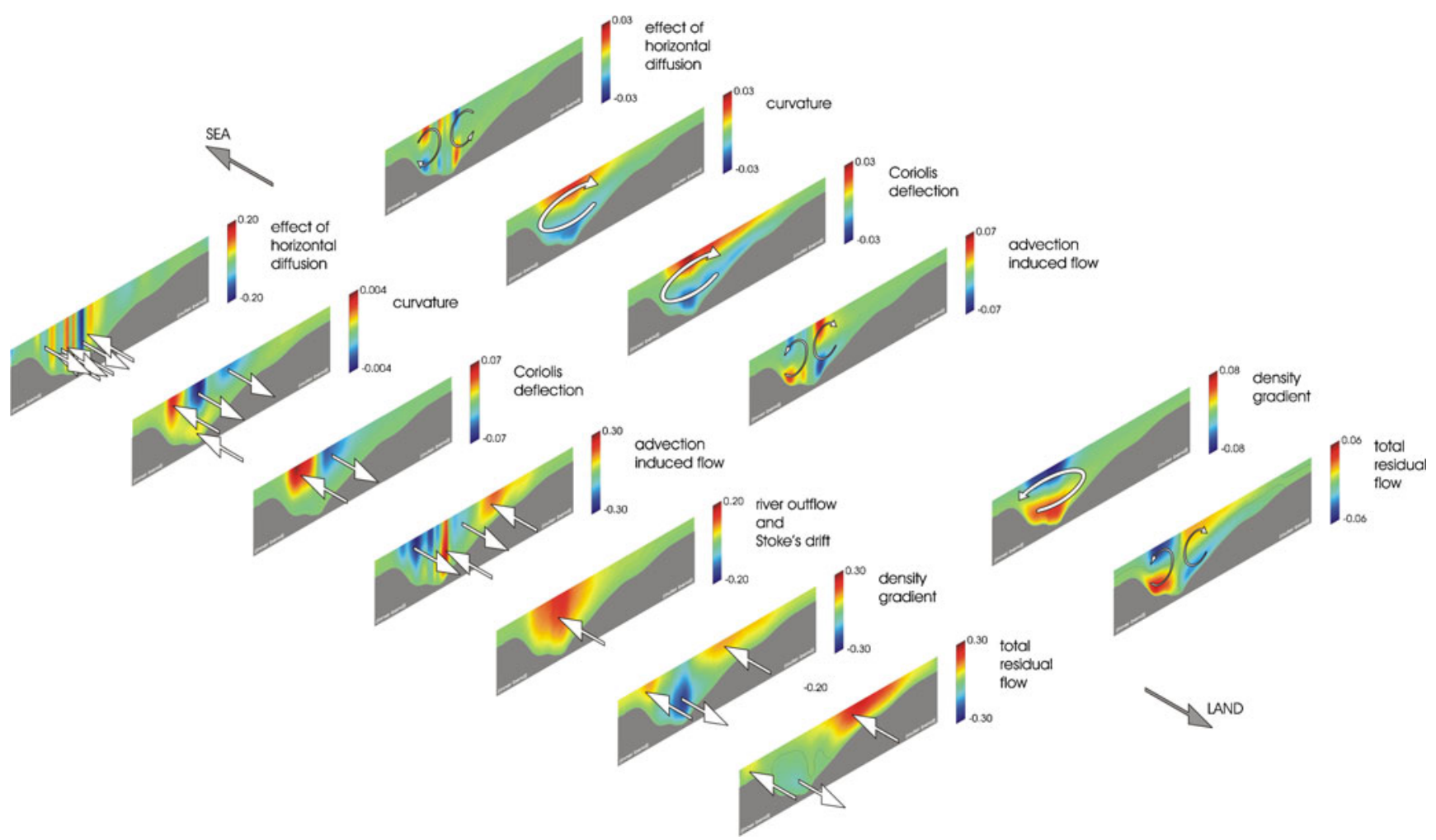

Fig. 7 Decomposition of the tide-residual flow computed assuming a parabolic eddy viscosity distribution. Lower left panels concern the along-channel flow and the cross-channel flow is given in the upper right panels. The total tide-residual flow is shown in the panels on the

In along-channel direction, the order of magnitude of flow velocities associated to contributions from river outflow and gravitational circulation is comparable to that of the total residual flow. Around the centre of the channel, these two mechanisms counteract each other, whereas the effect of gravitational circulation dominates at the comparatively shallow flanks. This is in agreement with the theoretical reasoning presented in "Appendix 5".

In cross-channel direction, curvature, Coriolis deflection and density gradient yield single circulation cells. When looking seaward, the circulations due to curvature and Coriolis deflection are clockwise. The effect of curvature is relatively weak as the radius of curvature is rather large (about $40 \mathrm{~km}$ ). The circulation related to the transverse density gradient is anti-clockwise, and it is attended with larger flow velocities than the other two. The contributions of curvature and Coriolis deflection to the along-channel tide-residual flow are relatively small.

The tide-residual advection-induced along-channel flow computed assuming a parabolic eddy viscosity distribution seems to be organised more or less in vertical strips with alternating flow direction (Fig. 7). The computational result based on a uniform eddy viscosity distribution (Fig. 8) shows a similar tendency, but it right. Warm colours indicate a flow towards the sea (for the alongchannel component) or to the right (for the transverse component). Flow velocities are given in metres per second

contains also some vertical variation. To some extent, these patterns can be recognized also in the corresponding forcings (Fig. 9a, b). In transverse direction, advection induces two opposing tide-residual circulations, one on either side of the deepest point of the channel and an upward flow in between. With a uniform eddy viscosity distribution, the flow velocities are about three times as large as in the case of a parabolic distribution. Furthermore, the computational results suggest a relation between the magnitude of the flow velocity and the transverse bottom steepness. Seen in transverse direction, the advection-induced tide-residual flow seems to increase in areas where the bottom steepens. This holds for both considered eddy viscosity distributions and for both along and cross-channel flow velocity components.

The effect of transverse diffusion is much alike that of advection. The flow induced in along-channel direction is organised more or less in vertical strips in which the flow is alternately seaward and landward, but with respect to number and size of these strips the resemblance is poor. In crosschannel direction, we find two opposing circulations on either side of the centre of the channel and a downward flow in between. The circulations found for advectioninduced flow act the other way round. 


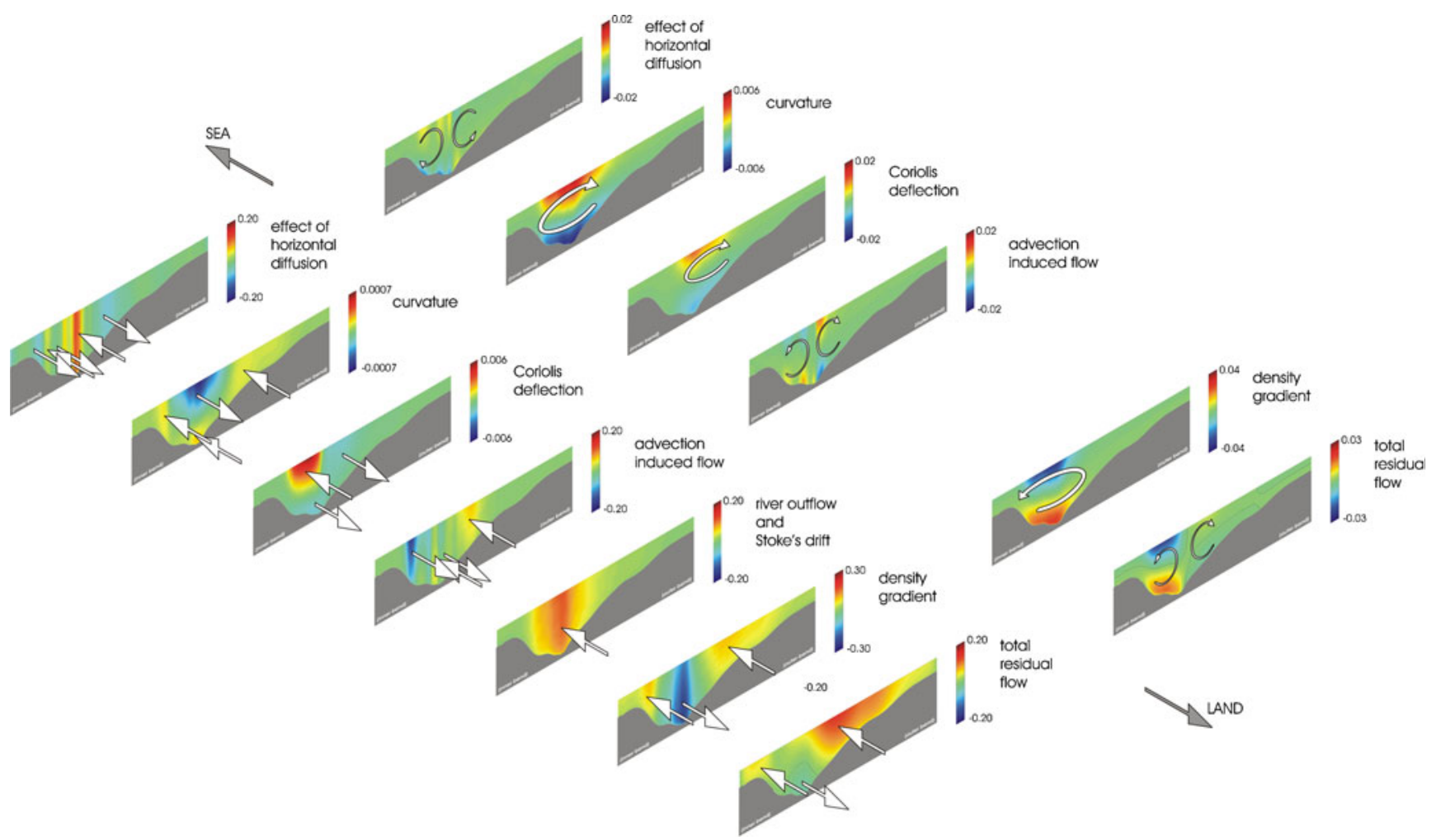

Fig. 8 Decomposition of the tide-residual flow computed assuming a uniform distribution of eddy viscosity over the vertical. Lower left panels concern the along-channel flow and the cross-channel flow is given in the upper right panels. The total tide-residual flow is shown in

\section{Analysis}

The computational results presented in Figs. 7 and 8 show for both considered types of eddy viscosity distribution that the contributions due to advection and horizontal diffusion to the total tide-residual flow are of the same order of magnitude as the total. Apparently, in the Chesapeake Bay transect studied, advection and transverse diffusion cannot be neglected in leading order. In addition, the computational results show that along the transect noticeable variations occur in their effects.

Fig. 9 Tide-residual along-channel advective forcing computed assuming a uniform eddy viscosity (a, c) and assuming a parabolic shape of the vertical distribution of eddy viscosity (b, d). Warm colours indicate a seaward force. a, $\mathbf{b}$ Results of computations in which advection is taken into account in leading order. That is not the case for the computations of which the results are shown in $\mathbf{c}$ and d a

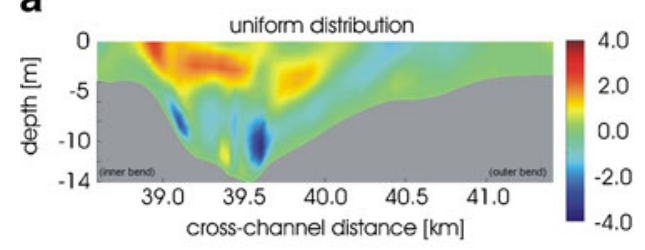

C

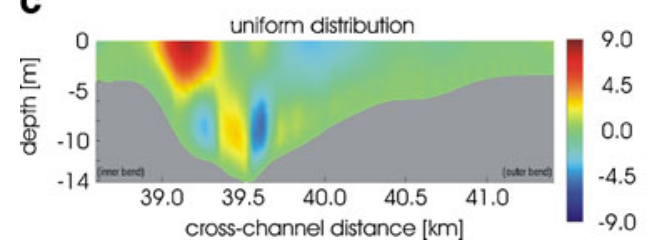

the panels on the right. Warm colours indicate a flow towards the sea (for the along-channel component) or to the right (for the transverse component). Flow velocities are given in metres per second

Motivated by this observation, we explore hereafter the possibility of expressing the relative importance of advection in terms of characteristics of the flow field, like Rossby and Stokes numbers. Such an expression may serve as a general aid in assessing whether advection can be assumed negligible in leading order and, hence, whether an idealized approach is adequate to describe and analyse the crosssectional flow pattern. Simultaneously, we assess the existence of a causal relation between local spatial variations in advective and diffusive forcing on the one hand and local transverse variations in bottom topography on the other

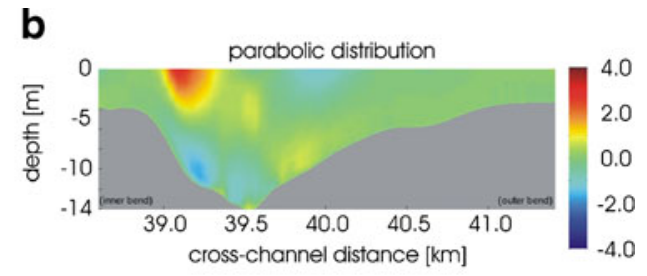

d

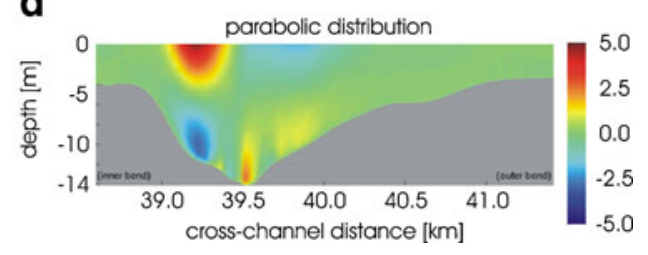


hand. We deploy the IM for these purposes as it provides a semi-analytical and therefore more transparent solution of its basic equations than the case-specific, numerical solutions obtained with the AM.

We use flow velocity profiles obtained with the IM to arrive at a first-order estimate of advective forcing. In this respect, we focus on the $M_{0}$ and $M_{2}$ components of a flowdriving force $d$, given by

$d(t)=d_{0}+d_{\mathrm{c}} \cos \omega t+d_{\mathrm{s}} \sin \omega t$

with $\omega$ the angular frequency of the semi-diurnal tidal constituent. This force can be related to either an along-channel density gradient, a transverse density gradient or an alongchannel surface slope. As outlined in "Appendix 3", the corresponding first-order estimate of along-channel advective forcing can be written as a linear combination of an $M_{0}$, an $M_{2}$ and an $M_{4}$ component. Denoting these components by $A_{0}$ (for the $M_{0}-M_{0}$ interaction), $A_{2}$ (for the $M_{0}-M_{2}$ interaction) and $A_{4}$ (for the $M_{2}-M_{2}$ interaction), the estimate becomes

$u_{y} \frac{\partial u_{x}}{\partial y}+u_{z} \frac{1}{h} \frac{\partial u_{x}}{\partial \sigma}=A_{0}(\sigma)+A_{2}(\sigma, t)+A_{4}(\sigma, t)$

(in which $x$ and $y$ are the along-channel and cross-channel coordinates, respectively, as used in the formulation of the IM in "Appendix 3").

The amplitudes of these components are given by

$$
\begin{aligned}
& A_{0}(\sigma)=g^{2} \gamma \beta^{3} \frac{1}{h} \frac{\mathrm{d} z_{\mathrm{b}}}{\mathrm{d} y}\left(d_{0}^{2}+\frac{d_{\mathrm{c}}^{2}+d_{\mathrm{s}}^{2}}{2}\right) \mu_{0}(\sigma) \\
& \widehat{A}_{2}(\sigma)=g^{2} \gamma \beta^{3} \frac{1}{h} \frac{\mathrm{d} z_{\mathrm{b}}}{\mathrm{d} y}\left(2 d_{0} \sqrt{d_{\mathrm{c}}^{2}+d_{\mathrm{s}}^{2}}\right) \mu_{2}(\sigma) \\
& \widehat{A}_{4}(\sigma)=g^{2} \gamma \beta^{3} \frac{1}{h} \frac{\mathrm{d} z_{\mathrm{b}}}{\mathrm{d} y}\left(\frac{d_{\mathrm{c}}^{2}+d_{\mathrm{s}}^{2}}{2}\right) \mu_{4}(\sigma)
\end{aligned}
$$

(^ denotes 'amplitude') in which $z_{\mathrm{b}}$ is the bottom level, $y$ the transverse coordinate and the $\mu$ are shape functions for the vertical distribution of the concerned amplitudes.

In the above expressions, the amplitudes $\widehat{A}$ are written as products of a scale factor and a shape function. These shape functions depend on the type of flow-driving mechanism and on the assumed vertical distribution of eddy viscosity. Examples of shape functions are given in Figs. 10 and 11. They concern a flow driven by an along-channel density gradient. Figure 10 contains the shape functions obtained for a uniformly distributed eddy viscosity, whereas Fig. 11 is based on a parabolic eddy viscosity profile.

In setting up these two figures, two contributions to the shape function $\mu_{0}$ have been discriminated. One of them is due to the $M_{0}-M_{0}$ interaction of the flow-driving force, whereas the other results from the $M_{2}-M_{2}$ interaction. Similar results are obtained for a flow driven by an alongchannel surface slope.
Although less apparent for the response to the $M_{0}-M_{0}$ interaction, all distribution functions presented in Figs. 10 and 11 show a tendency of decreasing order of magnitude with decreasing Ekman number. For a sufficiently small Ekman number, the relative importance of vertical diffusion vanishes and apart from an ever thinner bottom boundary layer, the horizontal flow becomes constant over the vertical. Combined with a zero net flow in cross-channel direction, this means that the transverse flow velocity tends towards zero. From continuity, it then follows that also the vertical velocity tends towards zero (at least in our model as we assume along-channel uniformity). Consequently, our first-order estimate of along-channel advective forcing decreases with decreasing Ekman number.

For a flow driven by an along-channel density gradient, the orders of magnitude of the shape functions $\mu_{0}, \mu_{2}$ and $\mu_{4}$ are much alike. This can be seen from Figs. 10 and 11. This holds also for a flow driven by an along-channel surface slope. With this property, an approximate scale of the first-order estimate of along-channel advective forcing can be written as

$u_{y} \frac{\partial u_{x}}{\partial y}+u_{z} \frac{1}{h} \frac{\partial u_{x}}{\partial \sigma} \sim g^{2} \gamma \beta^{3} \frac{1}{h} \frac{\mathrm{d} z_{\mathrm{b}}}{\mathrm{d} y} D_{\theta}^{2} \mu_{A}$

in which $\mu_{\mathrm{A}}$ is the order of magnitude of the shape functions and $D_{\theta}$ is a measure for the magnitude of the along-channel driving force, defined by

$D_{\theta}=d_{0}+\sqrt{d_{\mathrm{c}}^{2}+d_{\mathrm{s}}^{2}}$

This follows from Eqs. 14 and 15 if $\mu_{0}=\mu_{2}=\mu_{4}$. If this scale is small compared to that of other terms in the along-channel momentum balance, it is likely that along-channel advective forcing can be neglected in leading order. Such a comparison can be seen as an indicative test for the relative importance of this advective forcing. It can be elaborated below.

With the velocity scales

$u_{x} \sim-g \beta D_{\theta} \quad ; \quad u_{y} \sim-g \gamma \beta^{2} D_{\theta}$

(cf. Eq. 71 in "Appendix 3"), we obtain according to our IM the following scales for terms in the along-channel momentum balance:

- Inertia:

$$
\frac{\partial u_{x}}{\partial t} \sim-g \omega \beta \alpha_{t} D_{\theta}=-\mathrm{St}^{-1} \cdot g \alpha_{\mathrm{t}} D_{\theta} \quad ; \quad \alpha_{\mathrm{t}}=\frac{\sqrt{d_{\mathrm{c}}^{2}+d_{\mathrm{s}}^{2}}}{D_{\theta}}
$$

- Coriolis deflection:

$$
\gamma u_{y} \mu_{\mathrm{C}} \sim g(\gamma \beta)^{2} D_{\theta}=\mu_{\mathrm{C}} \mathrm{Ek}^{-2} \cdot g D_{\theta}
$$

- External forcing:

$$
-g D_{\theta}
$$

- Vertical diffusion: $-g D_{\theta}$

in which St is the Stokes number $\left(\mathrm{St}=(\omega \beta)^{-1}\right)$ and $\mathrm{Ek}$ is the Ekman number $\left(E k=(\gamma \beta)^{-1}\right)$. Furthermore, $\mu_{\mathrm{C}}$ is the order of 
Fig. 10 Vertical distribution of the first-order estimate of along-channel advective forcing as a function of Ekman number $(1 / \gamma \beta)$ and corresponding to a flow driven by an along-channel density gradient. Each panel shows the distribution function for a specific interaction between $M_{0}$ and $M_{2}$ components of the flow-driving force. A uniform eddy viscosity distribution is assumed

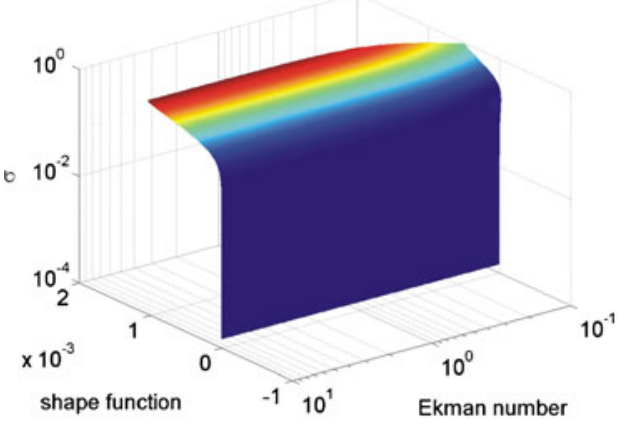

$M_{0}$ response to $M_{0}-M_{0}$ interaction

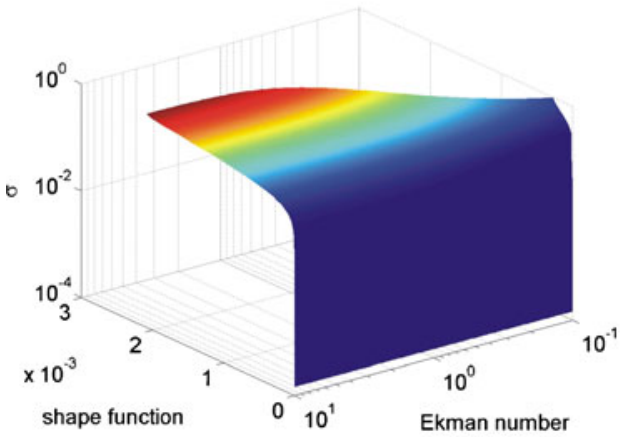

$M_{2}$ response to $M_{0}-M_{2}$ interaction

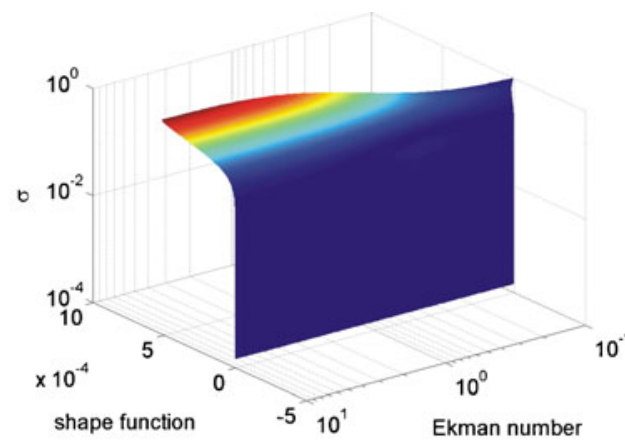

$M_{0}$ response to $M_{2}-M_{2}$ interaction

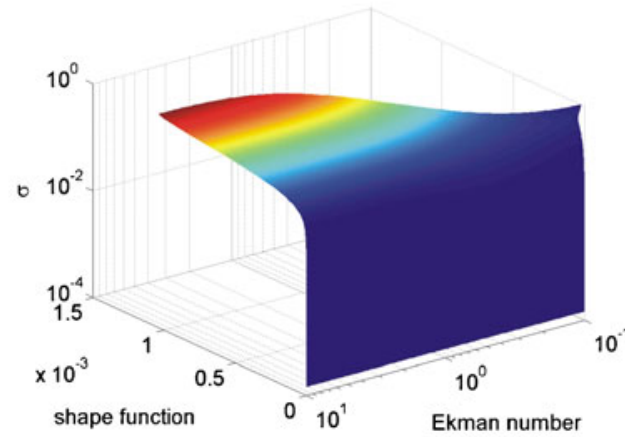

$M_{4}$ response to $M_{2}-M_{2}$ interaction magnitude corresponding to the shape function for the vertical distribution of Coriolis forcing. According to the IM, $\mu_{\mathrm{C}}=10^{-2}$ for a parabolic eddy viscosity distribution. Similar magnitudes for inertia and vertical diffusion are of order 1 (not mentioned explicitly in the above expressions).
For the scale of the first-order estimate of along-channel advection, we may write

- Advection:

$$
u_{y} \frac{\partial u_{x}}{\partial y}+u_{z} \frac{1}{h} \frac{\partial u_{x}}{\partial \sigma} \sim \mu_{\mathrm{A}} \cdot \mathrm{Ro} \cdot \mathrm{Ek}^{-2} \cdot g D_{\theta}
$$

Fig. 11 Vertical distribution of the first-order estimate of along-channel advective forcing as a function of Ekman number $(1 / \gamma \beta)$ and corresponding to a flow driven by an along-channel density gradient. Each panel shows the distribution function for a specific interaction between $M_{0}$ and $M_{2}$ components of the flow-driving force. A parabolic eddy viscosity distribution is assumed. White curves are zero contours
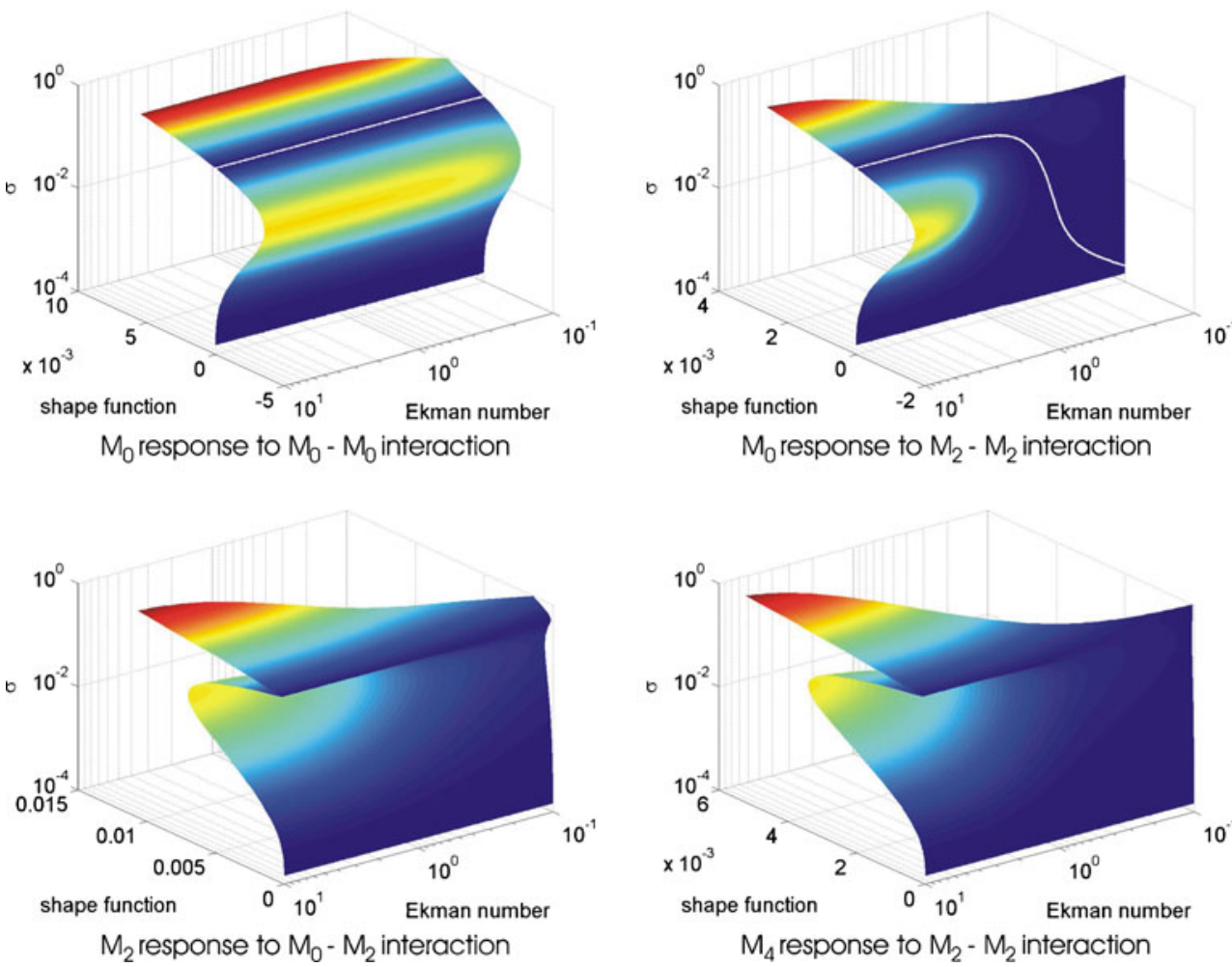
in which Ro is the Rossby number, defined by

$$
\text { Ro }=\frac{g \beta D_{\theta}}{\gamma L} \quad ; \quad L=\left(\frac{1}{h} \frac{\mathrm{d} \mathrm{z}_{\mathrm{b}}}{\mathrm{d} y}\right)^{-1}
$$

The numerator in the above expression is the scale of the along-channel velocity and $L$ in the denominator is a length scale that depends on the local water depth and the local transverse bottom slope.

Comparing the above scales and after dividing them by $g D_{\theta}$, it follows that along-channel advective forcing is likely to be relatively small if

$\mu_{\mathrm{A}} \cdot \mathrm{Ro} \cdot \mathrm{Ek}^{-2} \ll \max \left\{\alpha_{t} \cdot \mathrm{St}^{-1}, \mathrm{Ek}^{-2}, 1\right\}$

The above result forms an indicative test for the relative importance of along-channel advection in the case of a flow driven by a force acting in along-channel direction. It is indicative primarily as it is based on a first-order estimate of along-channel advection, but also as the shape of the vertical distribution of advective forcing is not taken into account, only its order of magnitude (parameter $\mu_{\mathrm{A}}$ ).

Figure 12 shows the scale factors involved in Eq. 20 for the Chesapeake Bay transect studied in the present paper. Apparently, the relative importance of advection (indicated with the solid line in the figure) varies along the cross section. Especially in the central part of the channel, it is not small compared to vertical diffusion. This indicates that advective forcing cannot be neglected in leading order.

Also apparent from Fig. 12 is that inertia dominates over vertical diffusion in the central, deeper part of the channel whereas the opposite is encountered in the adjacent comparatively shallow areas. This is consistent with the transverse variation of the relative Ekman depth (defined as $\sqrt{ }(2 \mathrm{Ek})$ and indicated also in Fig. 12). This relative depth is the smallest in the centre of the channel, indicating that in this area, the thickness of the bottom boundary layer is small compared to the local water depth. Away from this boundary layer, the flow pattern is dominated by inertia. In the shallow parts of the channel, on the other hand, the bottom boundary layer occupies a considerable part of the water column, and the flow pattern is influenced primarily by vertical diffusion.

According to our IM, the vertical distribution of eddy viscosity has a noticeable influence on the vertical structure of along-channel advective forcing, at least for a flow driven

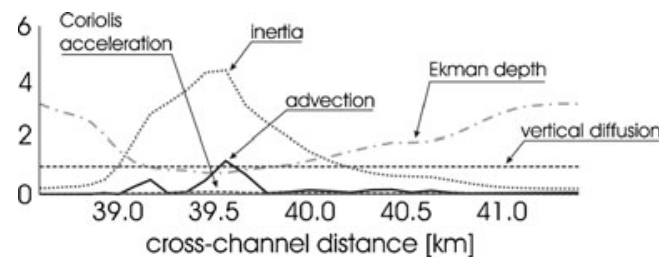

Fig. 12 Variation of scale factors for advection, inertia, vertical diffusion and Coriolis acceleration along the studied Chesapeake Bay transect. For comparison, the relative Ekman depth is added by an along-channel density gradient or surface slope. This becomes apparent by comparing Figs. 10 and 11 . In the case of a parabolic eddy viscosity distribution, the $M_{0}$ component of the advective forcing changes direction over the water column (upper panels in Fig. 12). For the Ekman number larger than about 1 , the amplitudes of the $M_{2}$ and $M_{4}$ components show two peaks: one in the vicinity of the bottom and one at the surface. This is consistent with the result obtained with the AM for the same type of eddy viscosity distribution and assuming that advection is negligible in leading order (see Fig. 9d). On the left-hand side of the centre of the channel, we see a landward forcing in the lower half of the water column and a seaward forcing in the upper half. A mirror image of this pattern is found on the right-hand side. This is straightforward as advective forcing is proportional to the transverse bottom slope and on either sides of the centre these slopes bear opposite signs.

For a uniform eddy viscosity, the $M_{0}$ component of along-channel advective forcing does not show a change of direction over the water column (upper panels in Fig. 10). Amplitudes of $M_{2}$ and $M_{4}$ components do not show a peak near the bottom but increase gradually towards the surface (lower panels in Fig. 10). This finding, based on our IM, does not seem very consistent with results obtained with the AM for the same eddy viscosity profile. Along-channel advective forcing derived from AM results includes changes of direction over the vertical and also in the horizontal at unexpected locations, considering the transverse bottom slope. Whether advection is or is not included in leading order in these AM computations hardly makes a difference (see Fig. 9a, c).

This seemingly anomalous behaviour can be explained from transverse diffusion. As outlined in "Appendix 4" on the basis of the IM, the along-channel forcing related to transverse diffusion can be seen as a combination of one part that scales with the local transverse bottom slope (similar to advective forcing) and another part that scales with the local transverse curvature of the bottom. This second part causes that bumps in the bottom lead to a local increase of the relative importance of horizontal diffusion. The relevance of this effect is assessed with an additional computation with the AM. It concerns a look-alike of the Chesapeake Bay transect with a rather smooth transverse bathymetry. Along-channel advective forcing derived from the results of this additional computation is given in Fig. 13 $\mathrm{b}$, together with the advective forcing found for the actual bathymetry (Fig. 13 a, identical to Fig. 9c). In both cases, advection is assumed negligible in leading order and eddy viscosity is distributed uniformly.

Apparently, the applied smoothing of the bottom profile substantially reduces the effect of horizontal diffusion. The tide-residual along-channel advective forcing found for the smoothed bottom profile (Fig. $13 \mathrm{~b}$ ) is far closer to the 


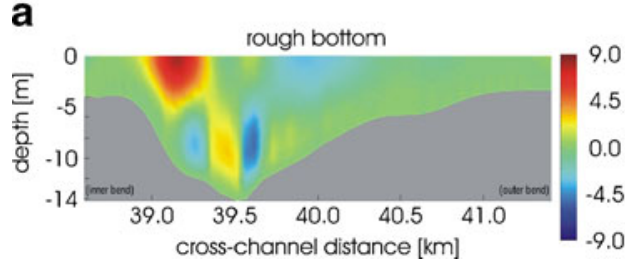

Fig. 13 Tide-residual along-channel advective forcing estimated from flow velocities computed assuming that advection is negligible in leading order. The left-hand panel shows the estimate obtained with

pattern expected on the basis of the semi-analytical solutions of the IM (Fig. 10a) than what is obtained for the actual bottom (Fig. 13 a). However, as horizontal diffusion is not suppressed completely, the agreement is not perfect.

That the spatial pattern of tide-residual advective forcing computed for a parabolic eddy viscosity profile agrees better with the outcome of the IM than that determined for a uniform eddy viscosity distribution suggests a larger relative importance of horizontal diffusion in the latter case. This probably has to do with the difference of the scale of eddy viscosity in the two cases. According to the semi-analytical expressions obtained with our IM, transverse diffusion is proportional to the depth-mean eddy viscosity, whereas along-channel advection is proportional to third power of that mean. For a parabolic eddy viscosity profile that mean is in our computations a factor of four larger than that for a uniformly distributed eddy viscosity. This indicates that the importance of transverse diffusion relative to that of alongchannel advective forcing is much larger for a uniform eddy viscosity profile than for a parabolic one.

\section{Discussion}

To study the relative importance of advection, we reconstruct advective forcing from flow velocities computed with the AM. Subsequently, we determine which part of the total flow can be assigned to this particular forcing. Results show that in the Chesapeake Bay case, advection is not negligible in leading order. In a tide-residual sense, advection-induced flow is not small compared to contributions of other driving mechanisms to the total flow pattern (see Figs. 7 and 8).

We assess the relevance of taking advection into account in leading order by reconstructing tide-residual advective forcing from computed flow velocities. We compare two reconstructions. In one of them, flow velocities are computed including advection, whereas in the other computation advection is neglected. The results of these reconstructions are presented in Fig. 9. Those concerning a presumed uniform eddy viscosity distribution (panels a and c in Fig. 9) are obscured by effects of horizontal diffusion and therefore less suitable for the intended comparison. Results obtained for a parabolic eddy viscosity profile (panels $b$ and $d$ in

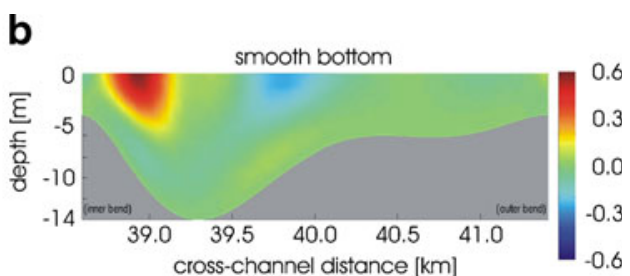

the actual cross-channel bathymetry of Chesapeake Bay, whereas an artificially smoothened profile underlies the estimate presented in the right-hand panel. Warm colours indicate a seaward force

Fig. 9) indicate that neglecting advection in leading order leads to a larger estimate of tide-residual along-channel advective forcing than when advection is included in the flow computations.

This difference may explain why Huijts et al. (2009) overestimate the magnitude and extent of the area around the lower part of the centre of the channel in which a residual landward flow occurs (their Fig. 2; also see Section 1 in the present paper). They compute the flow in the channel in two steps. The first step is a zero-order approximation in which advection is presumed negligible. Advective forcing is estimated from the so-obtained flow pattern and used subsequently as an external forcing in a first-order approximation. Our model results indicate that for the Chesapeake Bay case, such an approach leads to an overestimation of the influence of advection on the tideresidual along-channel flow pattern.

In essence, the approach followed by Huijts et al. (2009) is based on the hypothesis that the effect of advection can be described mathematically as a series expansion. Their elaboration of that series covers the first two terms only, which may be too little to reach sufficient convergence.

Semi-analytical expressions for first-order estimates of along-channel advective forcing obtained with our IM indicate an essential difference in the spatial pattern of that forcing, depending on the assumed eddy viscosity profile. For a flow driven by an along-channel surface slope or an along-channel density gradient, we find for a uniform eddy viscosity distribution that the advective forcing monotonously increases with height above the bottom. Model results concerning Chesapeake Bay presented by Huijts et al. (2009) bear the same property. Our theoretical result shows that this property is not case-specific but that it is inherent to a uniform eddy viscosity distribution.

Seen in cross-channel direction, however, the direction of the along-channel advective forcing may vary. The reason for this is that this forcing is proportional to the local transverse bottom slope. Hence, for a fairly simple channel geometry in which the bottom level increases monotonously with distance from the centre, the along-channel advective forcing is organised in two cells, one on either side of the centre of the channel. Within such a cell the magnitude of advective forcing may vary, but it has the same direction 
everywhere. However, the direction changes from one cell to the other. This is consistent with the findings of Huijts et al. (2009, their Fig. 9h).

For a parabolic eddy viscosity profile, on the other hand, our theoretical first-order estimate of along-channel advective forcing changes direction over the vertical (see Fig. 11). In the lower part of the water column, the forcing is directed opposite to that in the upper part. In the case of a channel geometry like the aforementioned fairly simple one, this leads to a transverse distribution of along-channel advective forcing consisting of four cells. Such a pattern can be recognized in Fig. 9d showing the first-order estimate of along-channel advective forcing derived from flow velocities computed with the AM assuming a parabolic eddy viscosity profile.

With the semi-analytical expressions for a first-order estimate of along-channel advective forcing obtained with our IM, we derive an indicative test to judge the relative importance of advection from orders of magnitude of alongchannel flow-driving mechanisms. This test is formulated in terms of common oceanographic index numbers, viz. the Ekman number, the Stokes number and the Rossby number. Deviant from what is used in most theoretical studies, we relate the length scale that is part of the Rossby number to a local geometrical property of the transverse channel profile instead of a global property. This ensues directly from the theoretical outcome of our IM, showing that along-channel advective forcing is proportional to the local transverse bottom slope and inversely proportional to the local water depth. Following this finding, we define the length scale to be used in the Rossby number as the ratio of water depth and transverse bottom slope. Hence, our test indicates the local relative importance of along-channel advection. Whether this advection should be taken into account in leading order in flow modelling depends on the locations and extent of areas in the transect where it is relatively important. This applies especially to studies based on a natural transect. In many theoretical studies on this subject, however, a schematised and smooth bottom profile is used, fully described by some continuous mathematical function. For such a profile, local and global properties may be strongly interrelated, so a global property may be as appropriate as a local one to reflect the relative importance of advection.

Transverse diffusion is not included in our IM, but its semianalytical outcome can be used to arrive at a first-order estimate of the associated forcing, similar to the way advective forcing is estimated. In the present study, this is elaborated for a flow driven by an along-channel surface slope and density gradient. The theoretical result shows that the magnitude of the diffusive forcing is directly related to the transverse bathymetry, in particular the local water depth, transverse bottom slope and transverse curvature of the bottom. This may be a source of numerical artefacts, for instance when a polynomial fit is used to describe a bottom profile. Such a fit may be perfect in the sense that it exactly reproduces observed bottom levels but simultaneously be attended with the introduction of bumps that do not exist in the physical bottom. In model computations, such bumps lead to an artificial amplification of the effect of transverse diffusion.

\section{Conclusion}

To study the importance of cross-sectional bathymetry and eddy viscosity parameterisation in modelling estuarine flow, we develop two models. One is an advanced numerical model that solves the full three-dimensional shallow water equations for a transect of a curved tidal channel, assuming alongchannel uniformity and also that water level variations are small compared to water depth (rigid-lid approximation). The other model is focused on idealized conditions in which advective processes and transverse diffusion can be neglected in leading order. For those conditions, this model provides semi-analytical solutions of the shallow water equations as well as for first-order estimates of along-channel advective forcing and transverse diffusive forcing. This second model is meant primarily as an aid in understanding and explaining results obtained with the numerical model.

First, we calibrate our numerical model to observations gathered in a transect of Chesapeake Bay. Second, we deploy this model to compute tide-residual flow using three parameterisations of eddy viscosity, all in combination with a no-slip bottom boundary condition: a parabolic vertical profile (used also in the calibration), a uniform distribution and a combination of a parabolic profile in the vicinity of the bottom and uniform higher up in the water column. The latter is used to simulate a partial-slip bottom boundary condition imposed at the level of transition from parabolic to uniform eddy viscosity. With respect to the computed tide-residual flow patterns, the uniform distribution and the combined form yield hardly any difference.

On average, assuming a uniform eddy viscosity distribution yields larger tide-residual flow velocities than in the case of a parabolic distribution over the water column. Corresponding spatial patterns, on the other hand, are similar to one another. For both parameterisations, advective and transverse diffusive processes have a noticeable effect on the tide-residual flow in the considered transect. Flow resulting from these processes contributes significantly to the total tidal residual in the concerned Chesapeake Bay transect. This holds for the along-channel flow component as well as for the transverse one. In earlier studies, advection and diffusion were presumed negligible in leading order, but that is not supported by our results.

In search for an explanation for the apparent significance of advection and diffusion-induced flow, we deploy our 
idealized model as it provides semi-analytical expressions for first-order estimates of along-channel advective and diffusive forcing. From these expressions, we find that the spatial pattern of along-channel advective forcing changes noticeably depending on whether a uniform or a parabolic eddy viscosity distribution is assumed. For a uniform eddy viscosity profile, the along-channel advective forcing does not change over the water column. In the case of a parabolic profile, on the other hand, the direction of the advective forcing in the lower part of the water column is opposite to that in the upper part. This holds in general. It is not a case-specific finding.

Furthermore, our idealized model shows that the magnitude of along-channel advective forcing scales with local transverse bottom slope and local water depth. From this finding, we conclude that judging whether or not alongchannel advective forcing should be included in leading order in flow computations is better based on local geometrical properties of the considered channel cross section than on global ones, like channel width.

The relative importance of transverse diffusion depends also on local properties of the channel cross section, in particular the curvature of the bottom profile. We therefore recommend to avoid artificial bumps, however small, in mathematical representations of bottom profiles to be used in flow models.

From our idealized model results, we conclude that the combination of transverse bottom topography and type of eddy viscosity parameterisation largely determines the spatial distribution of along-channel tide-residual advective forcing. In parts of the channel's transect where the bottom slope does not change sign, this forcing is organised in cells. Assuming a uniform eddy viscosity distribution leads to a single cell in the water column. When a parabolic eddy viscosity profile is assumed, on the other hand, two cells on top of each other occur with forcing in opposite directions. For an idealized transverse bottom profile, like a parabolic one, such cell structures occur at either side of the centre of the channel. They are each other's mirror image. This way, a uniform eddy viscosity distribution leads to two cells and a parabolic one to four cells in the cross section.

A more realistic, usually more bumpy transverse topography may lead to a larger number of cells in cross-channel direction as the transverse bottom slope changes sign more often. On top of that, the cell structure may be obscured by the effect of horizontal diffusion. That effect gains importance due to the bumps. The combined effect may be rather complex as can be seen in our numerical model output.

Throughout our study, we presume that eddy viscosity is time-invariant. Although the study has provided new insight into aspects of estuarine flow, this assumption may be too restrictive for various practically and scientifically relevant cases. Therefore, flow-dependent eddy viscosity will be addressed in a subsequent study.
Acknowledgements The investigation reported in the present paper is part of the research programme of the Delft University of Technology. The authors express their thanks to Dr. D. Fugate for allowing them to use his field data on Chesapeake Bay.

Open Access This article is distributed under the terms of the Creative Commons Attribution Noncommercial License which permits any noncommercial use, distribution, and reproduction in any medium, provided the original author(s) and source are credited.

\section{Appendix 1: Coordinate transformation and numerical method}

In the AM, flow velocity components and the transverse pressure gradient are solved numerically using a collocation method based on Chebyshev polynomials (see, e.g. Boyd 2000). In the physical domain, we use a non-orthogonal curvilinear grid. This domain is mapped onto a rectangular computational domain in such a way that seen in both directions, grid points in the computational domain coincide with collocation points (see Fig. 14). As we apply the rigidlid approximation, this mapping is time-invariant.

The coordinate system $(\zeta, \eta)$ is defined such that the physical domain (the channel's cross-section) is covered exactly by $\zeta \in[-1 ; 1]$ and $\eta \in[-1 ; 1]$. The bottom of the channel and the water surface coincide with $\eta=-1$ and $\eta=1$, respectively, whereas the two side walls correspond to $\zeta=-1$ and $\zeta=1$. Furthermore, curves of constant $\zeta$ appear in the physical domain as straight, vertical lines.

The relative height of lines of constant $\eta$ above the bottom does not change in transverse direction. As the bottom level varies across the channel, these lines are curved. As this curvature is taken into account fully in transforming the basic model Eqs. 1, 2 and 3 to the coordinate system $(\zeta, \eta)$ of the computational domain, numerical effects like artificial flow (see, e.g. Stelling and Van Kester 1994) will not occur.

The basic model equations are transformed to the $(\zeta, \eta)$ coordinate system by consistent and continued application of the general relations

$\frac{\partial}{\partial \xi}=\frac{\partial r}{\partial \xi} \frac{\partial}{\partial r}+\frac{\partial z}{\partial \xi} \frac{\partial \xi}{\partial z} \quad ; \quad \frac{\partial}{\partial \eta}=\frac{\partial r}{\partial \eta} \frac{\partial}{\partial r}+\frac{\partial z}{\partial \eta} \frac{\partial}{\partial z}$

Subsequently, the flow velocity components $u_{r}, u_{\theta}$ and $u_{z}$ are solved from the transformed model equations using a collocation method based on Chebyshev polynomials (see, e.g. Boyd 2000). The essence of this approach can be explained below. Velocity components are approximated by a linear combination of Chebyshev polynomials:

$$
\left(\begin{array}{c}
u_{r}(\xi, \eta, t) \\
u_{\theta}(\xi, \eta, t) \\
u_{z}(\xi, \eta, t)
\end{array}\right)=\sum_{k=0}^{K} \sum_{m=0}^{M}\left(\begin{array}{c}
X_{k m}(t) \\
Y_{k m}(t) \\
Z_{k m}(t)
\end{array}\right) T_{k}(\xi) T_{m}(\eta)
$$


Fig. 14 Non-orthogonal curvilinear grid in the physical domain and the corresponding rectilinear, orthogonal grid in the computational domain. Grid points in the latter domain are collocation points based on Chebyshev polynomials applied in two directions. To illustrate the spatial variation of resolution, four grid lines are coloured red. Those in the physical domain correspond to the ones in the computational domain

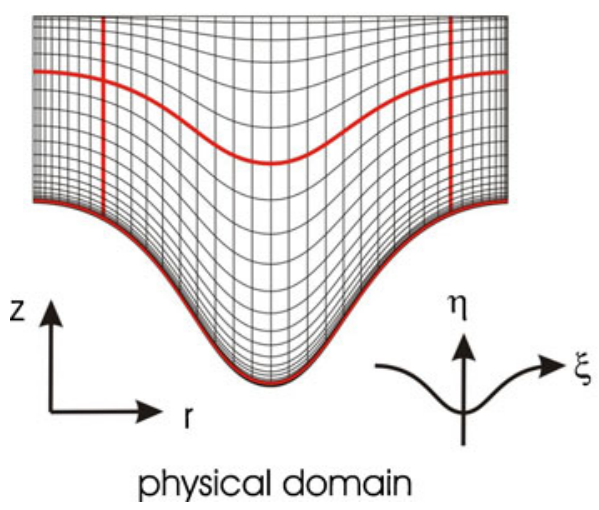

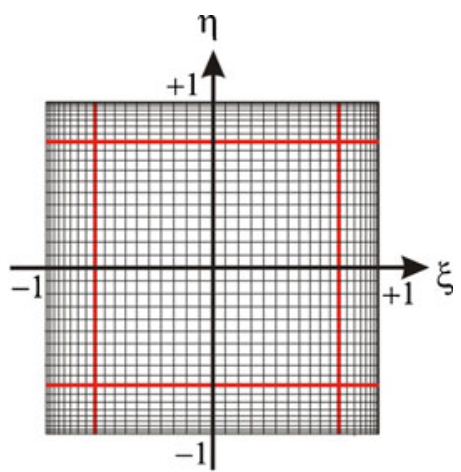

computational domain in which $T_{j}$ denotes a Chebyshev polynomial of order $j$. The $X, Y$ and $Z$ are time-dependent weight factors. They are determined such that series approximations of the velocities satisfy the transformed model equations exactly in the collocation points $\left(\zeta_{i}, \eta_{j}\right)$, with $i=0 \ldots K$ and $j=0 \ldots M$. In the computational domain, these points include the boundaries and the zeros-crossings of $\mathrm{d} T_{K} / \mathrm{d} \zeta$ for $\zeta$ and those of $\mathrm{d} T_{M} / \mathrm{d} \eta$ for $\eta$ (Lobatto grid, see, e.g. Boyd 2000). As the derivative of $T_{n}(n>0)$ contains $n-1$ zero-crossings, we have $(K+1)(M+1)$ collocation points. Evaluation of the transformed momentum and mass balances including corresponding boundary conditions in these points yields a set of $3(K+1)(M+1)$ equations. This is identical to the number of weight factors $X_{k m}, Y_{k m}$ and $Z_{k m}$. The set is therefore sufficient to solve these factors.

The along-channel surface slope $\partial \zeta / \partial \theta$ is chosen such that the model produces a prescribed time-dependent discharge. It is taken constant along the transect, but it may vary with time. The transverse surface slope $\partial \zeta / \partial r$ is computed by the model in accordance with the rigid-lid approximation. For each vertical grid line, it is determined such that Eq. 5 is satisfied.

The number and the spatial distribution of collocation points in the computational domain is fully determined once a choice has been made regarding the extent of the series expansions like Eq. 22 for the velocity components, i.e. once $K$ and $M$ have been set. Their distribution in the physical domain, on the other hand, can be influenced to some extent by manipulating the relation between the coordinate systems $(r, z)$ and $(\zeta, \eta)$, provided that in mapping the physical domain onto the computational domain correspondence between boundaries is maintained and that no folds occur. In our model, we use this freedom to achieve a comparatively high resolution near the bottom of the channel. This is necessary for a proper representation of the bottom boundary layer. In particular, the transformation from the vertical coordinate $z$ to $\eta$ is performed in two steps. The first step is a scaling of $z$ to $\sigma$, such that $\sigma=0$ at the bottom and $\sigma=1$ at the surface. This scaling depends on the location in the cross section of the channel. In the second step, a non-linear transformation of $\sigma$ to $\eta$ is applied to achieve a relatively high resolution near the bottom. An example of this transformation is shown graphically in Fig. 15.

The dots in Fig. 15 are situated on a curve given by

$\sigma=\sigma_{0}+\left(1-\sigma_{0}\right) s\left(\exp \left(\frac{\eta+1}{2} \ln \frac{1+s}{s}\right)-1\right)$

in which $\sigma_{0}$ is the relative height above the bottom at which the bottom boundary condition is applied (equal to $10^{-3}$ in the example in Fig. 15) and $s$ is a shape parameter. The vertical lines intersect the $\eta$ axis at collocation points. The distribution of these points in the interval $[-1 ; 1]$ is fixed. Where these vertical lines intersect the curve given by Eq. 23 determines the distribution of grid points between the level at which the bottom boundary condition is applied ( $\left.\sigma=\sigma_{0}\right)$ and the surface $(\sigma=1)$ in the physical domain. This distribution can be influenced by varying the shape parameter $s$. Choosing $s$ identical to $\sigma_{0}$ yields a logarithmic distribution. Decreasing the value of $s$ increases the resolution near the bottom at the expense of that in the upper part of the water column. In all model computations carried out for the present study, $s=10^{-4}$ has been used.

For integration in the time domain, we use a fully implicit finite difference approximation. This means that we approximate

$\left.\frac{\partial u}{\partial t}\right|_{t=t_{n+1}} \approx \frac{u^{(n+1)}-u^{(n)}}{t_{n+1}-t_{n}}$

in which the superscripts $(n+1)$ and $(n)$ refer to the time levels $t_{n+1}$ and $t_{n}$, respectively. In the model computations mentioned in the present paper, we use $t_{n+1}-t_{n}=$ $81 \mathrm{~s}$, so that one tidal cycle $(44,712 \mathrm{~s})$ is covered by exactly 552 time steps. Test computations have shown that reducing the time step size has negligible effect on model output. 


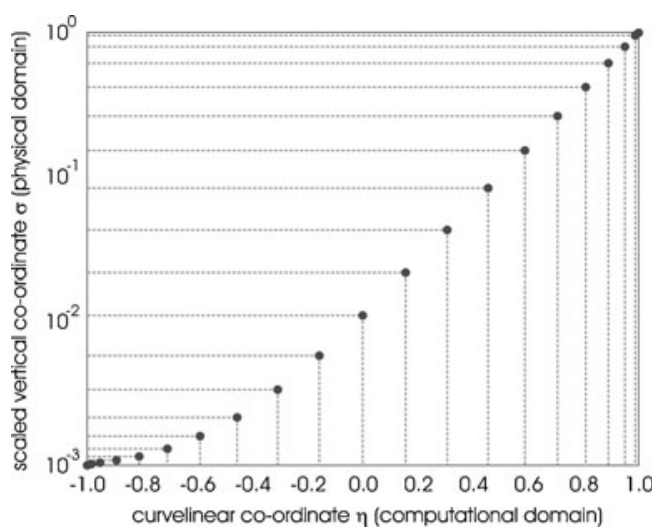

Fig. 15 Example of the transformation of the scaled vertical coordinate $\sigma$ in the physical domain to the coordinate $\eta$ in the computational domain. At the surface, $\sigma=1$ and $\eta=1$, whereas at the level at which the no-slip bottom boundary conditions is applied, $\eta=-1$ and, in this example, $\sigma=0.001$

With approximation Eq. 24, the momentum balance for the radial direction Eq. 1 can be written as

$\frac{u_{r}^{(n+1)}-u_{r}^{(n)}}{t_{n+1}-t_{n}}-\mathrm{N}_{r}\left(u_{r}^{(n+1)}, u_{\theta}^{(n+1)}, u_{z}^{(n+1)}, \xi, \eta, t_{n+1}\right)=0$

in which $N_{r}$ is an operator defined such that the above expression is identical to Eq. 1 into which the approximation Eq. 24 has been substituted. Similar expressions can be formulated for the other two basic model equations, but for transparency we focus on the above one in the present appendix. We interpret the left-hand side of Eq. 25 as a function of the velocity components at time level $t_{n+1}$ and in which the known velocity $u_{r}^{(n)}$ occurs as a parameter (with a prescribed value). We denote this function by $f_{r}$. After substitution of the series expansion (Eq. 22) for the three velocity components, we may express this as

$f_{r}\left(X_{k m}^{(n+1)}, Y_{k m}^{(n+1)}, Z_{k m}^{(n+1)} ; \xi, \eta, t_{n+1}\right)=0 \quad ; \quad\left\{\begin{array}{l}k=0 . . K \\ m=0 . . M\end{array}\right.$

The weight factors $X, Y$ and $Z$ for time level $t_{n+1}$ are found by solving the above equation at all collocation points simultaneously. As $f_{r}$ is non-linear in these factors, we use a Newton-Raphson iteration (see, e.g. Abramowitz and Stegun (1970)). In this respect, the $X, Y$ and $Z$ obtained for time level $t_{n}$ serve as first estimates. Successive corrections $\Delta X, \Delta Y$ and $\Delta Z$ to these estimates are obtained from

$f_{r}+\sum_{k=0}^{K} \sum_{m=0}^{M} \Delta X_{k m} \frac{\partial f_{r}}{\partial X_{k m}}+\sum_{k=0}^{K} \sum_{m=0}^{M} \Delta Y_{k m} \frac{\partial f_{r}}{\partial Y_{k m}}+\sum_{k=0}^{K} \sum_{m=0}^{M} \Delta Z_{k m} \frac{\partial f_{r}}{\partial Z_{k m}}=0$ evaluated for the current estimates of the weight factors and at all collocation points simultaneously, together with similar expressions derived from the momentum balance for the along-channel direction and from the continuity equation.

\section{Appendix 2: Contributions of individual driving forces to the flow pattern}

Horizontal flow velocity components $u_{\theta}$ and $u_{r}$ may be seen as a superposition of contributions that can be assigned to individual flow-driving forces:

$u_{\theta}=\sum_{i} u_{\theta, i} \quad ; \quad u_{r}=\sum_{i} u_{r, i}$

in which the index $i$ refers to a specific driving force and the $u_{\theta, i}$ and $u_{r, i}$ are the corresponding contributions. Computation of these contributions is performed in three consecutive steps. First, we compute the time- and space-dependent flow field with the AM. Subsequently, we discriminate between the driving forces mentioned in Table 2, and we use the results obtained with the AM to compute these forces. In the third step, the contributions $u_{\theta, i}$ and $u_{r, i}$ related to these forces are determined from

$$
\frac{\partial u_{\theta, i}}{\partial t}=S_{\theta, i}-g\left(\frac{1}{r} \frac{\partial \zeta}{\partial \theta}\right)_{i}+\frac{\partial}{\partial z}\left(v_{\mathrm{t}} \frac{\partial u_{\theta, i}}{\partial z}\right) ; \iint_{\substack{\text { cross } \\ \text { section }}} u_{\theta, i} \mathrm{~d} z \mathrm{~d} r=Q_{i}
$$

$$
\frac{\partial u_{r, i}}{\partial t}=S_{r, i}-g\left(\frac{\partial \zeta}{\partial r}\right)_{i}+\frac{\partial}{\partial z}\left(v_{\mathrm{t}} \frac{\partial u_{r, i}}{\partial z}\right) \quad ; \quad \int_{-h}^{0} u_{r, i} \mathrm{~d} z=0
$$

together with boundary conditions identical to those used in the AM:

$u_{\theta}(-h)=u_{r}(-h)=\left.\frac{\partial u_{\theta}}{\partial z}\right|_{0}=\left.\frac{\partial u_{r}}{\partial z}\right|_{0}=0$

In expressions 29 and $30, S_{\theta}$ and $S_{r}$ reflect the driving forces under consideration in along- and cross-channel direction, respectively. The along-channel surface slope is determined such that a total discharge $Q$ is obtained that corresponds to what the concerned driving force induces. The transverse slope, on the other hand, is computed in accordance with the rigid-lid approximation, also applied in the AM. The driving forces assessed in the present study are listed in Table 2. 
Table 2 Driving forces for which the contribution to the along- and cross-channel flow patterns is determined

\begin{tabular}{llll}
\hline Origin of driving force & $\begin{array}{l}\text { Description } \\
\text { (along-channel direction) }\end{array}$ & $\begin{array}{l}\text { Attended } \\
\text { (cross-channel direction) }\end{array}$ & $\begin{array}{l}\text { discharge } \\
\text { Advection }\end{array}$ \\
Coriolis & $S_{\theta}=-u_{r} \frac{\partial u_{\theta}}{\partial r}-u_{z} \frac{\partial u_{\theta}}{\partial z}$ & $S_{r}=-u_{r} \frac{\partial u_{r}}{\partial r}-u_{z} \frac{\partial u_{r}}{\partial z}$ & $Q=0$ \\
Along-channel density gradient & $S_{\theta}=-\gamma u_{r}$ & $S_{r}=\gamma u_{\theta}$ & $Q=0$ \\
Cross-channel diffusion & $S_{\theta}=\frac{z}{r} \frac{g}{\rho_{0}} \frac{\partial \rho}{\partial \theta}$ & $S_{r}=z \frac{g}{\rho_{0}} \frac{\partial \rho}{\partial r}$ & $Q=0$ \\
Curvature & $S_{\theta}=v_{h} \frac{1}{r} \frac{\partial}{\partial r}\left(r \frac{\partial u_{\theta}}{\partial r}\right)$ & $S_{r}=v_{\mathrm{h}} \frac{1}{r} \frac{\partial}{\partial r}\left(r \frac{\partial u_{r}}{\partial r}\right)$ & $Q=0$ \\
River discharge & $S_{\theta}=-\frac{u_{\theta} u_{r}}{r}$ & $S_{r}=\frac{u_{\theta}^{2}}{r}$ & $Q=0$ \\
\hline
\end{tabular}

\section{Appendix 3: Estimating advection with the idealized model}

The basic equations of the IM are given in Eqs. 6, 7, 8 and 9. With the complex notation

$u=u_{x}+i u_{y} \quad ; \quad i^{2}=-1$

we may write these equations like

$$
\begin{aligned}
& \frac{\partial u}{\partial t}+i \gamma u=-g \frac{h}{\rho_{0}}(1-\sigma)\left(\rho_{x}+i \rho_{y}\right)-g\left(\zeta_{x}+i \zeta_{y}\right)+\frac{1}{\beta} \frac{\partial}{\partial \sigma}\left(\varphi \frac{\partial u}{\partial \sigma}\right) \\
& u\left(\sigma_{0}\right)=\left.\frac{\partial u}{\partial \sigma}\right|_{\sigma=1}=0 \\
& \int_{\sigma_{0}}^{1} \operatorname{Im}\{u\} \mathrm{d} \sigma=0
\end{aligned}
$$

in which $\beta$ is defined in Eq. 8 and $x, y, \sigma$ are along-channel, cross-channel and scaled vertical coordinates, $\zeta_{x}, \zeta_{y}$ are along and cross-channel barotropic pressure gradients and $\rho_{x}, \rho_{y}$ are along and cross-channel density gradients.

Condition 34 results from application of the rigid-lid approximation. It says that the depth-averaged flow in cross-channel direction equals zero at all times. The transverse surface slope $\zeta_{y}$ is chosen such that this condition 34 is met. Other flow-driving forces $\left(\rho_{x}, \rho_{y}\right.$ and $\left.\zeta_{x}\right)$ are prescribed and taken constant in cross-channel direction. In particular, we consider driving forces composed of a steady component and a harmonically varying one. For each force, this is written as the dot-product of two vectors:

$\zeta_{x}=\underline{\zeta}_{x} \bullet \underline{t} \quad ; \quad \rho_{x}=\underline{\rho}_{x} \bullet \underline{t} \quad ; \quad \rho_{y}=\underline{\rho}_{y} \bullet \underline{t} \quad ; \quad \underline{t}=\left(\begin{array}{c}1 \\ \cos \omega t \\ \sin \omega t\end{array}\right)$

The $\underline{\xi}_{y}, \underline{\rho}_{x}$ and $\underline{\rho}_{y}$ are real vectors representing the amplitudes of the $M_{0}$ and $M_{2}$ tidal components (with $\omega$ the angular frequency of $M_{2}$ ) of the along-channel surface slope, the along-channel and cross-channel density gradients, respectively. For instance, if

$$
\underline{\zeta}_{x}=\left(\begin{array}{c}
a_{0} \\
a_{\mathrm{c}} \\
a_{\mathrm{s}}
\end{array}\right)
$$

then

$$
\begin{aligned}
\zeta_{x} & =\underline{\zeta}_{x} \bullet \underline{t} \\
& =a_{0}+a_{\mathrm{c}} \cos \omega t+a_{\mathrm{s}} \sin \omega t \\
& =a_{0}+\sqrt{a_{\mathrm{c}}^{2}+a_{\mathrm{s}}^{2}} \sin (\omega t+\vartheta) \quad ; \quad \tan \vartheta=\frac{a_{\mathrm{c}}}{a_{\mathrm{s}}}
\end{aligned}
$$

with $\vartheta$ the phase angle of the $M_{2}$ component and $\sqrt{ }\left(a_{c}{ }^{2}+a_{\mathrm{s}}{ }^{2}\right)$ its amplitude. With Eqs. 35, 36 and 37, the basic model equation can be written as

$$
\begin{aligned}
\frac{\partial u}{\partial t}+i \gamma u & =-g \frac{h}{\rho_{0}}(1-\sigma)\left(\underline{\rho}_{x}+i \underline{\rho}_{y}\right) \bullet \underline{t}-g\left(\underline{\zeta}_{x}+i \underline{\zeta}_{y}\right) \bullet \underline{t}+\frac{1}{\beta} \frac{\partial}{\partial \sigma}\left(\varphi \frac{\partial u}{\partial \sigma}\right) ; \\
u\left(\sigma_{0}\right) & =\left.\frac{\partial u}{\partial \sigma}\right|_{\sigma=1}=0
\end{aligned}
$$

As this equation is linear in $u$, we may expect a solution of the form

$u=\underline{u} \bullet \underline{t}$

in which the complex vector $\underline{u}$ contains the amplitudes of the $M_{0}$ and $M_{2}$ tidal components of the flow velocity components in a similar way as Eqs. 36 and 37. To find this vector, we use the eigenfunction expansion

$\underline{u}(\sigma, t)=\sum_{m=1}^{M} f_{m}(\sigma) \underline{u}_{m}(t)$

in which the $\underline{u}_{m}$ contain time-dependent weight factors and the $f_{m}$ are vertical shape functions derived from the eigenvalue problem

$$
\frac{d}{\mathrm{~d} \sigma}\left(\varphi \frac{\mathrm{d} f_{m}}{\mathrm{~d} \sigma}\right)+\lambda_{m} f_{m}=0 \quad ; \quad f_{m}\left(\sigma_{0}\right)=\left.\frac{\mathrm{d} f_{m}}{\mathrm{~d} \sigma}\right|_{\sigma=1}=0
$$

where $\lambda_{m}$ are eigenvalues (see, e.g. Davies, 1987). The advantage of this choice lies in the similarity of the homogeneous boundary conditions imposed on the shape functions and the physical boundary conditions that apply to the horizontal velocity components. Due to this similarity, the expansion (Eq. 40) by definition meets those physical conditions. That is convenient as after substitution of the expansion into the basic model Eq. 38, the term reflecting vertical diffusion takes the form of a linear combination of shape functions. The explicit occurrence of vertical 
derivatives vanishes in this process. This necessitates an alternative imposition of the physical boundary conditions, which is found in the present study in the definition of the shape functions.
With the expansion, we may write the terms reflecting inertia, Coriolis deflection and vertical exchange of momentum in the basic model Eq. 33 as a linear combination of shape functions:

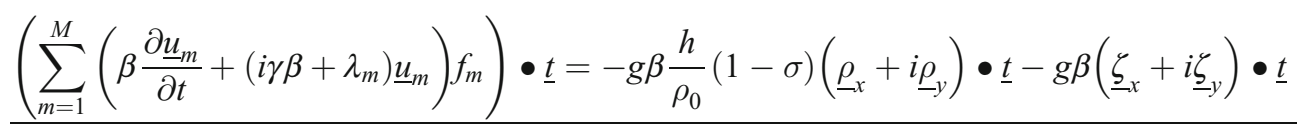

Subsequently, we apply the Galerkin technique to optimise the weight factors $\underline{u}_{m}$ (see, e.g. Boyd 2000). As the $f_{m}$ form a complete set of mutually orthogonal functions, the series expansion (Eq. 40) yields an exact solution if $M \rightarrow \infty$. For practical reasons, however, we truncate the series at $M=40$. A property of the Galerkin technique is that the error that remains after truncation cannot be expressed as a linear combination of the included shape function. In that sense, it yields an optimisation of the weights of those shape functions. Computational experiments on the convergence of the expansion have shown that for the considered flow conditions, $\underline{u}_{40}$ is at least 10 orders of magnitude smaller than $\underline{u}_{1}$.

Using Eq. 35, we may write Eq. 42 as

$$
\left(\sum_{m=1}^{M} f_{m} \mathbf{E}_{m} \underline{u}_{m}\right) \bullet \underline{t}=-g \beta \frac{h}{\rho_{0}}(1-\sigma)\left(\underline{\rho}_{x}+i \underline{\rho}_{y}\right) \bullet \underline{t}-g \beta\left(\underline{\zeta}_{x}+i \underline{\zeta}_{y}\right) \bullet \underline{t} \quad ; \quad \mathbf{E}_{m}=\left(\begin{array}{ccc}
i \gamma \beta+\lambda_{m} & 0 & 0 \\
0 & i \gamma \beta+\lambda_{m} & \omega \beta \\
0 & -\omega \beta & i \gamma \beta+\lambda_{m}
\end{array}\right)
$$

Note that apart from the eigenvalue $\lambda_{m}$, matrix $\mathrm{E}_{m}$ contains the Ekman number $\gamma \beta$ and the Stokes number $\omega \beta$. Application of the Galerkin technique and taking the orthogonality of the $f_{m}$ into account yields the following set of equations for the weight factors $\underline{u}_{m}$ :

$$
\mathbf{E}_{m} \underline{u}_{m}=-g \beta \frac{h}{\rho_{0}} \alpha_{1, m}\left(\underline{\rho}_{x}+i \underline{\rho}_{y}\right)-g \beta \alpha_{0, m}\left(\underline{\zeta}_{x}+i \underline{\zeta}_{y}\right) \quad ; \quad m=1 . . M
$$

in which

$\alpha_{k, m}=\frac{\int_{\sigma_{0}}^{1}(1-\sigma)^{k} f_{m} \mathrm{~d} \sigma}{\int_{\sigma_{0}}^{1} f_{m}^{2} \mathrm{~d} \sigma}$

By substituting condition Eq. 34 into Eq. 44, we may eliminate the transverse surface slope $\underline{\xi}_{y}$. This results in

$\underline{u}_{m}=-g \beta \frac{h}{\rho_{0}}\left(\mathbf{X}_{m} \underline{\rho}_{x}+i \mathbf{Y}_{m} \underline{\rho}_{y}\right)-g \beta \mathbf{Z}_{m} \underline{\zeta}_{x}$

in which

$$
\begin{aligned}
& \mathbf{X}_{m}=\mathbf{E}_{m}^{-1}\left(\alpha_{1, m} \mathbf{I}-i \alpha_{0, m} \operatorname{Re}\left\{\mathbf{D}_{0}\right\}^{-1} \operatorname{Im}\left\{\mathbf{D}_{1}\right\}\right) \\
& \mathbf{Y}_{m}=\mathbf{E}_{m}^{-1}\left(\alpha_{1, m} \mathbf{I}-\alpha_{0, m} \operatorname{Re}\left\{\mathbf{D}_{0}\right\}^{-1} \operatorname{Re}\left\{\mathbf{D}_{1}\right\}\right) \\
& \mathbf{Z}_{m}=\mathbf{E}_{m}^{-1}\left(\alpha_{0, m} \mathbf{I}-i \alpha_{0, m} \operatorname{Re}\left\{\mathbf{D}_{0}\right\}^{-1} \operatorname{Im}\left\{\mathbf{D}_{0}\right\}\right)
\end{aligned}
$$

with

$$
\mathbf{D}_{k}=\sum_{m=1}^{M} \alpha_{k, m} \mathbf{E}_{m}^{-1} F_{m} \quad ; \quad F_{m}=\int_{\sigma_{0}}^{1} f_{m} \mathrm{~d} \sigma
$$

and $I$ is a unit matrix of rank 3 .

Combination of Eq. 46 with Eq. 40 yields

$$
u(\sigma, t)=\sum_{m=1}^{M} f_{m}(\sigma)\left(-g \beta \frac{h}{\rho_{0}}\left(\mathbf{X}_{m} \underline{\rho}_{x}+i \mathbf{Y}_{m} \underline{\rho}_{y}\right)-g \beta \mathbf{Z}_{m} \underline{\zeta}_{x}\right) \bullet \underline{t}
$$

which is the zero-order approximation of the flow velocity profile for prescribed forcing ( $M_{0}$ and $M_{2}$ components of along-channel surface slope as well as along and crosschannel density gradients).

In our IM, along-channel advective forcing and crosschannel diffusion are assumed negligible in leading order. However, flow profiles computed as described above are used to arrive at first-order estimates of these quantities. Hereafter, we focus on advection. Diffusion is the subject of "Appendix 4".

For the estimate of advection, the vertical flow velocity $u_{z}$ is required. This flow component is derived from continuity and the bottom boundary condition according to

$$
\left.\begin{array}{l}
\frac{1}{h} \frac{\partial u_{z}}{\partial \sigma}+\frac{\partial \operatorname{Im}(u)}{\partial y}=0 \\
u_{z}\left(\sigma_{0}\right)=0
\end{array}\right\} \rightarrow \frac{1}{h} u_{z}=-\int_{\sigma_{0}}^{\sigma} \frac{\partial}{\partial y} \operatorname{Im}\{u(s)\} \mathrm{d} s
$$


Combination of this result with the rigid-lid approximation (which leads to Eq. 34) and the no-slip bottom boundary condition consistently yields $u_{z}(1)=0$.

To obtain the cross-channel derivative $\partial u / \partial y$, we consider a curvilinear, non-orthogonal coordinate system $(\psi, \sigma)$ similar to what is used in the AM (see Fig. 16: $\psi$ connects points of equal $\sigma$ along the transect). The coordinate $\psi$ is chosen such that $\partial \psi / \partial y=1$ and the vertical coordinate is defined by

$\sigma=\frac{z-z_{\mathrm{b}}}{h}$

with $z_{\mathrm{b}}$ bottom level elevation. As we apply the rigid-lid approximation, transverse variations in water depth $h$ and bottom level elevation $z_{\mathrm{b}}$ are equally large, but bear opposite signs $\left(z_{\mathrm{b}}=-h\right)$. Hence, $\frac{\mathrm{d} \sigma}{\mathrm{d} y}=-(1-\sigma) \frac{1}{h} \frac{\mathrm{d} z_{\mathrm{b}}}{\mathrm{d} y}$

With this property of the coordinate system, we may write

$\frac{\partial \bullet}{\partial y}=\frac{\partial \psi}{\partial y} \frac{\partial \bullet}{\partial \psi}+\frac{\partial \sigma}{\partial y} \frac{\partial \bullet}{\partial \sigma}=\frac{\partial \bullet}{\partial \psi}-(1-\sigma) \frac{1}{h} \frac{\mathrm{d} z_{\mathrm{b}}}{\mathrm{d} y} \frac{\partial \bullet}{\partial \sigma}$

Hence, combining Eq. 35 with eigenfunction expansion Eq. 40 gives

$\frac{\partial u}{\partial y}=\sum_{m=1}^{M}\left(f_{m} \frac{\partial \underline{u}_{m}}{\partial \psi}-\frac{1}{h} \frac{\mathrm{d} z_{\mathrm{b}}}{\mathrm{d} y}(1-\sigma) \frac{\mathrm{d} f_{m}}{\mathrm{~d} \sigma} \underline{u}_{m}\right) \bullet \underline{t}$

Substituting this derivative into Eq. 50 yields

$\frac{1}{h} u_{z}=\frac{1}{h} \underline{u}_{z} \bullet \underline{t}=-\sum_{m=1}^{M}\left(\frac{\partial \operatorname{Im}\left\{\underline{u}_{m}\right\}}{\partial \psi} \int_{\sigma_{0}}^{\sigma} f_{m}(s) \mathrm{d} s-\operatorname{Im}\left\{\underline{u}_{m}\right\}\left((1-\sigma) f_{m}+\int_{\sigma_{0}}^{\sigma} f_{m}(s) \mathrm{d} s\right) \frac{1}{h} \frac{\mathrm{d} z_{\mathrm{b}}}{\mathrm{d} y}\right) \bullet \underline{t}$

in which $\underline{u}_{z}$ is a real vector reflecting the amplitudes of the $M_{0}$ and $M_{2}$ tidal components of the vertical velocity (cf. Eq. 39).

To compute the derivative of the weight factors in the above expression, we write

$\frac{\partial \underline{u}_{m}}{\partial \psi}=\frac{\mathrm{d} \beta}{\mathrm{d} \psi} \frac{\partial \underline{u}_{m}}{\partial \beta}=\frac{\mathrm{d} \beta}{\mathrm{d} y} \frac{\partial \underline{u}_{m}}{\partial \beta}$

(note that $\beta$ does not vary with $\sigma$ ).

In many analytical models, it is assumed that the depthaveraged eddy viscosity scales with the water depth to some

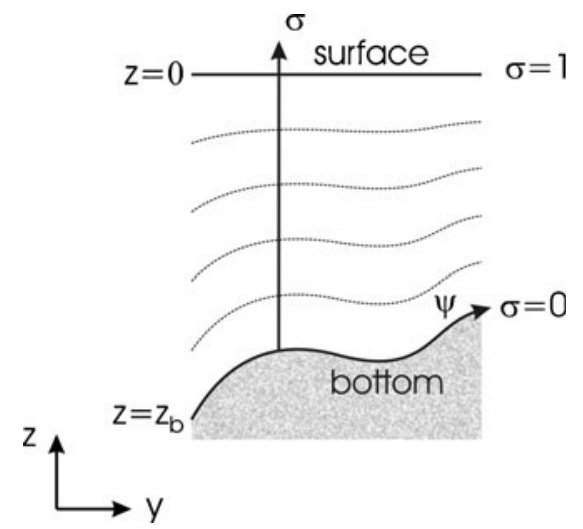

Fig. 16 Definition of the curvilinear, non-orthogonal coordinate system $(\psi, \sigma)$ used in the IM. $\psi$ connects points of equal $\sigma$ along the transect power $p$, where $p$ is of order 1. Friedrichs and Hamrick (1996) reason that for well-mixed conditions, $\bar{v}_{\mathrm{t}}$ scales with $h$, so that $p=1$. Alternatively, assuming resemblance with plane shear flow leads to $p=3 / 2$. In the case of a uniform eddy viscosity distribution, on the other hand, $p=0$. In any such case, it holds that

$\frac{\mathrm{d} \beta}{\mathrm{d} y}=\beta(p-2) \frac{1}{h} \frac{\mathrm{d} z_{\mathrm{b}}}{\mathrm{d} y}$

Elaboration of the derivative with respect to $\beta$ in Eq. 56 is a matter of consistent application of the chain rule to Eq. 46. We start by calculating

$$
\frac{\mathrm{d} \mathbf{E}_{m}}{\mathrm{~d} \beta}=\left(\begin{array}{ccc}
i \gamma & 0 & 0 \\
0 & i \gamma & \omega \\
0 & -\omega & i \gamma
\end{array}\right) \quad ; \quad \frac{\mathrm{d} \mathbf{D}_{k}}{\mathrm{~d} \beta}=-\sum_{m=1}^{M} \alpha_{k, m} F_{m} \mathbf{E}_{m}^{-2} \frac{\mathrm{d} \mathbf{E}_{m}}{\mathrm{~d} \beta}
$$

and resulting in

$$
\begin{aligned}
\frac{\partial \underline{u}_{m}}{\partial \psi} & =-\frac{1}{h} \frac{\mathrm{d} z_{\mathrm{b}}}{\mathrm{d} y} g \beta\left((p-3) \mathbf{X}_{m}+(p-2) \beta \frac{\mathrm{d} \mathbf{X}_{m}}{\mathrm{~d} \beta}\right) \frac{h}{\rho_{0}} \underline{\rho}_{x} \\
& -\frac{1}{h} \frac{\mathrm{d} z_{\mathrm{b}}}{\mathrm{d} y} g \beta\left((p-3) \mathbf{Y}_{m}+(p-2) \beta \frac{\mathrm{d} \mathbf{Y}_{m}}{\mathrm{~d} \beta}\right) \frac{h}{\rho_{0}} i \rho_{y} \\
& -\frac{1}{h} \frac{\mathrm{d} z_{\mathrm{b}}}{\mathrm{d} y} g \beta\left((p-2) \mathbf{Z}_{m}+(p-2) \beta \frac{\mathrm{d} \mathbf{Z}_{m}}{\mathrm{~d} \beta}\right) \underline{\zeta}_{x}
\end{aligned}
$$


in which

$$
\begin{aligned}
\frac{\mathrm{d} \mathbf{X}_{m}}{\mathrm{~d} \beta} & =-\mathbf{E}_{m}^{-1} \frac{\mathrm{d} \mathbf{E}_{m}}{\mathrm{~d} \beta} \mathbf{X}_{m}+i \alpha_{0, m} \mathbf{E}_{m}^{-1} \operatorname{Re}\left\{\mathbf{D}_{0}\right\}^{-1}\left(\frac{\mathrm{d} \operatorname{Re}\left\{\mathbf{D}_{0}\right\}}{\mathrm{d} \beta} \operatorname{Re}\left\{\mathbf{D}_{0}\right\}^{-1} \operatorname{Im}\left\{\mathbf{D}_{1}\right\}-\frac{\mathrm{d} \operatorname{Im}\left\{\mathbf{D}_{1}\right\}}{\mathrm{d} \beta}\right) \\
\frac{\mathrm{d} \mathbf{Y}_{m}}{\mathrm{~d} \beta} & =-\mathbf{E}_{m}^{-1} \frac{\mathrm{d} \mathbf{E}_{m}}{\mathrm{~d} \beta} \mathbf{Y}_{m}+\alpha_{0, m} \mathbf{E}_{m}^{-1} \operatorname{Re}\left\{\mathbf{D}_{0}\right\}^{-1}\left(\frac{\mathrm{d} \operatorname{Re}\left\{\mathbf{D}_{0}\right\}}{\mathrm{d} \beta} \operatorname{Re}\left\{\mathbf{D}_{0}\right\}^{1} \operatorname{Re}\left\{\mathbf{D}_{1}\right\}-\frac{\mathrm{d} \operatorname{Re}\left\{\mathbf{D}_{1}\right\}}{\mathrm{d} \beta}\right) \\
\frac{\mathrm{d} \mathbf{Z}_{m}}{\mathrm{~d} \beta} & =-\mathbf{E}_{m}^{-1} \frac{\mathrm{d} \mathbf{E}_{m}}{\mathrm{~d} \beta} \mathbf{Z}_{m}+i \alpha_{0, m} \mathbf{E}_{m}^{-1} \operatorname{Re}\left\{\mathbf{D}_{0}\right\}^{-1}\left(\frac{\mathrm{d} \operatorname{Re}\left\{\mathbf{D}_{0}\right\}}{\mathrm{d} \beta} \operatorname{Re}\left\{\mathbf{D}_{0}\right\}^{-1} \operatorname{Im}\left\{\mathbf{D}_{0}\right\}-\frac{\mathrm{d} \operatorname{Im}\left\{\mathbf{D}_{0}\right\}}{\mathrm{d} \beta}\right)
\end{aligned}
$$

Summarizing the above, semi-analytical expressions have been obtained for the horizontal flow velocity components $u$ (with Eq. 49), the vertical flow velocity component $u_{z}$ (with Eq. 55) and the transverse derivative of the horizontal flow velocity components (with Eqs. 54, 59 and 60). With these expressions, the mentioned velocity components and attended derivatives can be determined for a prescribed density gradient and along-channel surface slope. Each of these expressions applies to a single location along the channel's transect. Combining results for all locations yields the crosssectional flow pattern. These results apply to the idealized situation in which advection can be assumed negligible in leading order and in which external forcing (density gradient and along-channel surface slope) are composed of an $M_{0}$ and an $M_{2}$ component only.

With this result, our first-order estimate of the alongchannel advective forcing becomes

$$
\begin{aligned}
\operatorname{Im}\{u\} \frac{\partial \operatorname{Re}\{u\}}{\partial y}+u_{z} \frac{1}{h} \frac{\partial \operatorname{Re}\{u\}}{\partial \sigma} & =(\operatorname{Im}\{\underline{u}\} \bullet \underline{t})\left(\frac{\partial \operatorname{Re}\{\underline{u}\}}{\partial y} \bullet \underline{t}\right)+\left(\underline{u}_{z} \bullet \underline{t}\right)\left(\frac{1}{h} \frac{\partial \operatorname{Re}\{\underline{u}\}}{\partial \sigma} \bullet \underline{t}\right) \\
& =A_{0}(\sigma)+A_{2}(\sigma, t)+A_{4}(\sigma, t)
\end{aligned}
$$

In which $A_{0}, A_{2}$ and $A_{4}$ are the $M_{0}, M_{2}$ and $M_{4}$ components of the along-channel advective forcing, respectively.

By writing

$\operatorname{Re}\{\underline{u}\}=\left(\begin{array}{l}u_{x, 0} \\ u_{x, \mathrm{c}} \\ u_{x, \mathrm{~s}}\end{array}\right) \quad ; \quad \operatorname{Im}\{\underline{u}\}=\left(\begin{array}{l}u_{y, 0} \\ u_{y, \mathrm{c}} \\ u_{y, \mathrm{~s}}\end{array}\right) \quad ; \quad \underline{u_{z}}=\left(\begin{array}{l}u_{z, 0} \\ u_{z, \mathrm{c}} \\ u_{z, \mathrm{~s}}\end{array}\right)$

(cf. Eq. 36), these components can be written as

$$
\begin{aligned}
A_{0}= & u_{y, 0} \frac{\partial u_{x, 0}}{\partial y}+\frac{1}{2}\left(u_{y, \mathrm{c}} \frac{\partial u_{x, \mathrm{c}}}{\partial y}+u_{y, \mathrm{~s}} \frac{\partial u_{x, \mathrm{~s}}}{\partial y}\right)+u_{z, 0} \frac{1}{h} \frac{\partial u_{x, 0}}{\partial \sigma} \quad \text { 63) } \\
& +\frac{1}{2}\left(u_{z, \mathrm{c}} \frac{1}{h} \frac{\partial u_{x, \mathrm{c}}}{\partial \sigma}+u_{z, \mathrm{~s}} \frac{1}{h} \frac{\partial u_{x, \mathrm{~s}}}{\partial \sigma}\right) \\
A_{2}= & +\left(u_{y, 0} \frac{\partial u_{x, \mathrm{c}}}{\partial y}+u_{y, \mathrm{c}} \frac{\partial u_{x, 0}}{\partial y}+u_{z, 0} \frac{1}{h} \frac{\partial u_{x, \mathrm{c}}}{\partial \sigma}+u_{z, \mathrm{c}} \frac{1}{h} \frac{\partial u_{x, 0}}{\partial \sigma}\right) \cos \omega t \\
= & +\left(u_{y, 0} \frac{\partial u_{x, \mathrm{~s}}}{\partial y}+u_{y, \mathrm{~s}} \frac{\partial u_{x, 0}}{\partial y}+u_{z, 0} \frac{1}{h} \frac{\partial u_{x, \mathrm{~s}}}{\partial \sigma}+u_{z, \mathrm{~s}} \frac{1}{h} \frac{\partial u_{x, 0}}{\partial \sigma}\right) \sin \omega t
\end{aligned}
$$

$$
\begin{aligned}
A_{4} & =+\frac{1}{2}\left(u_{y, \mathrm{c}} \frac{\partial u_{x, \mathrm{c}}}{\partial y}-u_{y, \mathrm{~s}} \frac{\partial u_{x, \mathrm{~s}}}{\partial y}+u_{z, \mathrm{c}} \frac{1}{h} \frac{\partial u_{x, \mathrm{c}}}{\partial \sigma}-u_{z, \mathrm{~s}} \frac{1}{h} \frac{\partial u_{x, \mathrm{~s}}}{\partial \sigma}\right) \cos 2 \omega t \\
& =+\frac{1}{2}\left(u_{y, \mathrm{c}} \frac{\partial u_{x, \mathrm{~s}}}{\partial y}+u_{y, \mathrm{~s}} \frac{\partial u_{x, \mathrm{c}}}{\partial y}+u_{z, \mathrm{c}} \frac{1}{h} \frac{\partial u_{x, \mathrm{~s}}}{\partial \sigma}+u_{z, \mathrm{~s}} \frac{1}{h} \frac{\partial u_{x, \mathrm{c}}}{\partial \sigma}\right) \sin 2 \omega t
\end{aligned}
$$

First-order estimates of transverse diffusion can be obtained in a similar fashion. This is outlined in "Appendix 4".
Semi-analytical solutions for flow profiles obtained with the IM can be written as a product of a shape function and a scale factor. The velocity profile for a flow driven by an along-channel surface slope, for instance, reads

$u=-g \beta\left(\sum_{m=1}^{M} f_{m} \mathbf{Z}_{m} \underline{\zeta}_{x}\right) \bullet \underline{t}$

(see Eq. 49). In this expression, $-g \beta \underline{\xi}_{x}$ can be designated as a scale factor and $\Sigma f_{m} Z_{\mathrm{m}}$ as a shape function. Both contain three elements: one corresponding to the $M_{0}$ component of the response to the flow-driving force whereas the other two are related to the amplitude and phase of the $M_{2}$ response. We may take this one step further by looking closer at $Z_{m}$. From Eqs. 47 and 60, it can be seen that this matrix as well as its derivative with respect to $\beta$ is proportional to $E_{m}{ }^{-1}$. Evaluation of this inverse matrix reveals that its imaginary part scales with $\gamma \beta$. Its real part does not bear this property:

$\operatorname{Re}\left\{\mathbf{E}_{m}^{-1}\right\}=\left(\begin{array}{ccc}\frac{\lambda_{m}}{\lambda_{m}^{2}+(\gamma \beta)^{2}} & 0 & 0 \\ 0 & \lambda_{m} \frac{\lambda_{m}^{2}-3(\gamma \beta)^{2}+(\omega \beta)^{2}}{d} & -\omega \beta \frac{\lambda_{m}^{2}-(\gamma \beta)^{2}+(\omega \beta)^{2}}{d} \\ 0 & \omega \beta \frac{\lambda_{m}^{2}-(\gamma \beta)^{2}+(\omega \beta)^{2}}{d} & \lambda_{m} \frac{\lambda_{m}^{2}-3(\gamma \beta)^{2}+(\omega \beta)^{2}}{d}\end{array}\right)$ 
$\operatorname{Im}\left\{\mathbf{E}_{m}^{-1}\right\}=\gamma \beta\left(\begin{array}{ccc}\frac{-1}{\lambda_{m}^{2}+(\gamma \beta)^{2}} & 0 & 0 \\ 0 & \frac{3 \lambda_{m}^{2}-(\gamma \beta)^{2}+(\omega \beta)^{2}}{d} & -\frac{2 \lambda_{m}(\gamma \beta)(\omega \beta)^{2}}{d} \\ 0 & \frac{2 \lambda_{m}(\gamma \beta)(\omega \beta)^{2}}{d} & \frac{3 \lambda_{m}^{2}-(\gamma \beta)^{2}+(\omega \beta)^{2}}{d}\end{array}\right)$

with

$d=\left(\lambda_{m}^{2}-(\gamma \beta)^{2}+(\omega \beta)^{2}\right)^{2}+4 \lambda_{m}^{2}(\gamma \beta)^{2}$

As a result,

$\operatorname{Re}\{u\} \sim-g \beta \underline{\zeta}_{x} \quad ; \quad \operatorname{Im}\{u\} \sim-g \gamma \beta^{2} \underline{\zeta}_{x}$

A similar result is obtained for a flow driven by an alongchannel density gradient. For a cross-channel density gradient, we find

$\operatorname{Re}\{u\} \sim-g \gamma \beta^{2} \frac{h}{\rho_{0}} \underline{\rho}_{y} \quad ; \quad \operatorname{Im}\{u\} \sim-g \beta \frac{h}{\rho_{0}} \underline{\rho}_{y}$

Using Eqs. 49 and 59, the transverse derivative of the velocity profile can be written as the product of a scale factor and a shape function in a similar fashion. That holds also for the vertical velocity component $u_{z}$ and for $\partial u / \partial \sigma$. These scale factors are summarized in Table 3 for flow driven by either an along-channel density gradient, a transverse density gradient or an along-channel surface slope.

We use these scale factors to arrive at a general expression for the first-order estimate of along-channel advective forcing. For this purpose, we consider a flow induced by the $M_{0}$ and $M_{2}$ components of some mechanism. Like we did in expressions Eqs. 36 and 37 for the along-channel surface slope, we represent the amplitudes of these two harmonic components by a vector $\underline{d}$, defined by

$\underline{d}=\left(\begin{array}{c}d_{0} \\ d_{\mathrm{c}} \\ d_{\mathrm{s}}\end{array}\right)$

This vector stands for either $\left(h / \rho_{0}\right) \underline{\rho}_{x}$ or $\left(h / \rho_{0}\right) \underline{\rho}_{y}$ or $\underline{\xi}_{x}$. With this vector and the scale factors given in Table 3 , the amplitudes of the components $A_{0}, A_{2}$ and $A_{4}$ of the alongchannel advective forcing can be written as

$$
\begin{aligned}
& A_{0}(\sigma)=g^{2} \gamma \beta^{3} \frac{1}{h} \frac{\mathrm{d} z_{b}}{\mathrm{~d} y}\left(d_{0}^{2}+\frac{d_{\mathrm{c}}^{2}+d_{\mathrm{s}}^{2}}{2}\right) \mu_{0}(\sigma) \\
& \widehat{A}_{2}(\sigma)=g^{2} \gamma \beta^{3} \frac{1}{h} \frac{\mathrm{d} z_{b}}{\mathrm{~d} y}\left(2 d_{0} \sqrt{d_{\mathrm{c}}^{2}+d_{\mathrm{s}}^{2}}\right) \mu_{2}(\sigma) \\
& \widehat{A}_{4}(\sigma)=g^{2} \gamma \beta^{3} \frac{1}{h} \frac{\mathrm{d} z_{b}}{\mathrm{~d} y}\left(\frac{d_{\mathrm{c}}^{2}+d_{\mathrm{s}}^{2}}{2}\right) \mu_{4}(\sigma)
\end{aligned}
$$

(^ stand for 'amplitude') in which the $\mu$ are shape functions for the vertical distribution of the concerned amplitudes. Similar expressions are obtained for combinations of flowdriving forces. They have the scale factor $g^{2} \gamma \beta^{2}\left(\mathrm{~d} z_{\mathrm{b}} / \mathrm{d} y\right) / h$ in common. We use this property in the present study to judge indicatively the relative importance of this type of forcing.

\section{Appendix 4: Estimating cross-channel diffusive forcing with the idealized model}

As our IM is basically a single point model, it does not include transverse diffusion in leading order. A firstorder estimate diffusive forcing can be obtained, however, in the same fashion as the first-order estimate of advective forcing is derived in "Appendix 3". Hereafter, this is demonstrated for a flow driven by an alongchannel surface slope. The procedure for a density gradient-driven flow is a similar one.

According to the IM, the zero-order approximation of a flow driven by an along-channel surface slope is given by

$u=-g \beta\left(\sum_{m=1}^{M} f_{m} \mathbf{Z}_{m} \underline{\boldsymbol{\zeta}}_{x}\right) \bullet \underline{t}$

(see Eq. 46). The corresponding estimate of cross-channel diffusive forcing is given by

$v_{\mathrm{h}} \frac{\partial^{2} u}{\partial y^{2}}$

We elaborate Eq. 75 assuming that the flow-driving force does not vary in transverse direction. Furthermore, we use the curvilinear, non-orthogonal $(\psi, \sigma)$ coordinate system introduced in "Appendix 3" and shown in Fig. 16. The firstorder transverse derivative in this system is given in Eq. 53. The second-order derivative reads

\begin{tabular}{|c|c|c|c|c|}
\hline Flow-driving force & $\operatorname{Im}\{u\}$ & $\frac{\partial \operatorname{Re}\{u\}}{\partial y}$ & $\frac{1}{h} u_{z}$ & $\frac{\partial \operatorname{Re}\{u\}}{\partial \sigma}$ \\
\hline $\begin{array}{l}\text { Along-channel density gradient } \\
\text { Transverse density gradient } \\
\text { Along-channel surface slope }\end{array}$ & $\begin{array}{l}-g \gamma \beta^{2} \frac{h}{\rho_{0}} \underline{\rho}_{x} \\
-g \beta \frac{h}{\rho_{0}} \underline{\rho}_{y} \\
-g \gamma \beta^{2} \underline{\zeta}_{x}\end{array}$ & $\begin{array}{l}-g \beta \frac{1}{h} \frac{\mathrm{d} z_{\mathrm{b}}}{\mathrm{d} y} \frac{h}{\rho_{0}} \rho_{x} \\
-g \gamma \beta^{2} \frac{1}{h} \frac{\mathrm{d} z_{\mathrm{b}}}{\mathrm{d} y} \frac{h}{\rho_{0}} \rho_{y} \\
-g \beta \frac{1}{h} \frac{\mathrm{d} z_{\mathrm{b}}}{\mathrm{d} y} \underline{\zeta}_{x}\end{array}$ & $\begin{array}{l}-g \gamma \beta^{2} \frac{1}{h} \frac{\mathrm{d} z_{\mathrm{b}}}{\mathrm{d} y} \frac{h}{\rho_{0}} \rho_{x} \\
-g \beta \frac{1}{h} \frac{\mathrm{d} z_{\mathrm{b}}}{\mathrm{d} y} \frac{h}{\rho_{0}} \frac{\rho}{y} \\
-g \gamma \beta^{2} \frac{1}{h} \frac{\mathrm{d} z_{\mathrm{b}}}{\mathrm{d} y} \underline{\zeta}_{x}\end{array}$ & $\begin{array}{l}-g \beta \frac{h}{\rho_{0}} \underline{\rho}_{x} \\
-g \gamma \beta^{2} \frac{h}{\rho_{0}} \underline{\rho}_{y} \\
-g \beta \underline{\zeta}_{x}\end{array}$ \\
\hline
\end{tabular}

Table 3 Scale factors for flow velocities and related quantities 


$$
\begin{aligned}
\frac{\partial^{2} \bullet}{\partial y^{2}}= & \frac{\partial \psi}{\partial y}\left(\frac{\partial}{\partial \psi}\left(\frac{\partial \psi}{\partial y}\right) \frac{\partial \bullet}{\partial \psi}+\frac{\partial \psi}{\partial y} \frac{\partial^{2} \bullet}{\partial \psi^{2}}+\frac{\partial}{\partial \psi}\left(\frac{\partial \sigma}{\partial y}\right) \frac{\partial \bullet}{\partial \sigma}+\frac{\partial \sigma}{\partial y} \frac{\partial^{2} \bullet}{\partial \psi \partial \sigma}\right) \\
& +\frac{\partial \sigma}{\partial y}\left(\frac{\partial}{\partial \sigma}\left(\frac{\partial \psi}{\partial y}\right) \frac{\partial \bullet}{\partial \psi}+\frac{\partial \psi}{\partial y} \frac{\partial^{2} \bullet}{\partial \sigma \partial \psi}+\frac{\partial}{\partial \sigma}\left(\frac{\partial \sigma}{\partial y}\right) \frac{\partial \bullet}{\partial \sigma}+\frac{\partial \sigma}{\partial y} \frac{\partial^{2} \bullet}{\partial \sigma^{2}}\right)
\end{aligned}
$$

With definition Eq. 51 of $\sigma$, it follows that

$$
\begin{gathered}
\frac{\partial \sigma}{\partial y}=-(1-\sigma) \frac{1}{h} \frac{\mathrm{d} z_{\mathrm{b}}}{\mathrm{d} y} \frac{\partial}{\partial \psi}\left(\frac{\partial \sigma}{\partial y}\right)=-(1-\sigma)\left(\left(\frac{1}{h} \frac{\mathrm{d} z_{\mathrm{b}}}{\mathrm{d} y}\right)^{2}+\frac{1}{h} \frac{d^{2} z_{\mathrm{b}}}{\mathrm{d} y^{2}}\right) \\
\frac{\partial}{\partial \sigma}\left(\frac{\partial \sigma}{\partial y}\right)=\frac{1}{h} \frac{\mathrm{d} z_{\mathrm{b}}}{\mathrm{d} y} \frac{\partial}{\partial \psi}\left(\frac{\partial \psi}{\partial y}\right)=0
\end{gathered}
$$

so that the derivative in Eq. 67 can be written as

$$
\begin{aligned}
\frac{\partial^{2} \bullet}{\partial y^{2}} & =\frac{\partial^{2} \bullet}{\partial \psi^{2}}+\left(\frac{1}{h} \frac{\mathrm{d} z_{\mathrm{b}}}{\mathrm{d} y}\right)^{2}\left(-2(1-\sigma) \frac{\partial \bullet}{\partial \sigma}+(1-\sigma)^{2} \frac{\partial^{2} \bullet}{\partial \sigma^{2}}\right) \\
& -\frac{1}{h} \frac{\mathrm{d} z_{\mathrm{b}}}{\mathrm{d} y} 2(1-\sigma) \frac{\partial^{2} \bullet}{\partial \sigma \partial \psi}-(1-\sigma) \frac{1}{h} \frac{d^{2} z_{\mathrm{b}}}{\mathrm{d} y^{2}} \frac{\partial \bullet}{\partial \sigma}
\end{aligned}
$$

Substitution of the tidal velocity $u$ given in Eq. 74 into the above expression for the second-order derivative yields for the diffusive forcing

$$
\begin{aligned}
& v_{\mathrm{h}} \frac{\partial^{2}}{\partial y^{2}}\left(-g \beta \sum_{m=1}^{M} f_{m} \mathbf{Z}_{m} \underline{\zeta}_{x}\right)=-g v_{\mathrm{h}} \beta\left(\frac{1}{h} \frac{\mathrm{d} z_{\mathrm{b}}}{\mathrm{d} y}\right)^{2} \sum_{m=1}^{M}\left(f_{m} \mathbf{H}_{m}+(1-\sigma) \frac{\mathrm{d} f_{m}}{\mathrm{~d} \sigma} \mathbf{J}_{m}+(1-\sigma)^{2} \frac{d^{2} f_{m}}{\mathrm{~d} \sigma^{2}} \mathbf{Z}_{m}\right) \underline{\zeta}_{x} \\
& -g v_{\mathrm{h}} \beta \frac{1}{h} \frac{d^{2} z_{\mathrm{b}}}{\mathrm{d} y^{2}} \sum_{m=1}^{M}\left(f_{m} \mathbf{K}_{m}-(1-\sigma) \frac{\mathrm{d} f_{m}}{\mathrm{~d} \sigma} \mathbf{Z}_{m}\right) \underline{\zeta}_{x}
\end{aligned}
$$

in which

$$
\begin{aligned}
& \mathbf{H}_{m}=(p-2)(p-1) \mathbf{Z}_{m}+(p-2)(3 p-5) \beta \frac{\mathrm{d} \mathbf{Z}_{m}}{\mathrm{~d} \beta}+(p-2) \beta^{2} \frac{d^{2} \mathbf{Z}_{m}}{\mathrm{~d} \beta^{2}} \\
& \mathbf{J}_{m}=-2(p-1) \mathbf{Z}_{m}+-2(p-2) \beta \frac{\mathrm{d} \mathbf{Z}_{m}}{\mathrm{~d} \beta} \\
& \mathbf{K}_{m}=(p-2) \mathbf{Z}_{m}+(p-2) \beta \frac{\mathrm{d} \mathbf{Z}_{m}}{\mathrm{~d} \beta}
\end{aligned}
$$

To find the transverse diffusive forcing related to an along-channel density gradient, $Z_{m}$ and $\underline{\zeta}_{x}$ in Eqs. 79 and 80 should be replaced by $X_{m}$ and $\underline{\rho}_{x}$, respectively, or by $Y_{m}$ and $\underline{\rho}_{y}$ in the case of a transverse density gradient. With this result, we have obtained semi-analytical expressions for the transverse diffusive forcing for prescribed horizontal density gradient and along-channel surface slope.

\section{Appendix 5: Eddy viscosity scale as calibration parameter}

In the present investigation, we evaluate the performance of our AM by comparing computed and observed tideresidual along-channel flow. In this respect, the scale of the eddy viscosity is used as a calibration parameter. The observations show a landward residual flow in the lower part of the water column around the deep centre of the channel's cross section and a seaward flow almost everywhere else (see Fig. 4a). The zero velocity contour in this figure marks the boundary between the areas with opposite flow directions. This contour looks somewhat like the cross section of a bell. In the calibration of the $\mathrm{AM}$, we have seen that the scale of the eddy viscosity influences the size of this bell. It becomes smaller with increasing eddy viscosity and vice versa.

In the present appendix, we use our IM to explain this tide-residual flow pattern and how the AM can be tuned to properly reproduce this pattern by varying the scale of the eddy viscosity. For this purpose, we schematise the flow pattern in the cross section to a superposition of three contributions. One of them is related to a harmonically varying along-channel surface slope. In our linear IM, this contribution does not generate a residual. Another contribution is due to a steady river discharge into the channel. It is driven by a timeinvariant along-channel surface slope chosen such that it produces a prescribed discharge $Q$. The third contribution is a steady gravitational circulation with a landward component in the lower part of the water column and a seaward component in the upper part. Averaged over the channel's cross section, however, there is no net flow. As our interest is due to tide-residuals only, we may limit this discussion to the latter two contributions.

Defining $u_{\text {riv }}$ as that part of the horizontal flow velocity components that can be ascribed to the river discharge, it must hold that

$Q=\int_{\text {transect }} h \int_{\sigma_{0}}^{1} \operatorname{Re}\left\{u_{\mathrm{riv}}\right\} \mathrm{d} \sigma \mathrm{d} y$

As this $u_{\text {riv }}$ is generated by an along-channel surface slope, we may write, according to the IM, 
$u_{\text {riv }}=-g \beta \sum_{m=1}^{M} f_{m} \mathbf{Z}_{m} \underline{\zeta}_{x}$

(cf. Eq. 46, with matrix $Z_{m}$ defined in Eq. 47). As we focus on steady conditions, only the upper element of vector $\underline{\xi}_{x}$ is non-zero. This means that for the product $Z_{m} \underline{\xi}_{x}$, only the left column of $Z_{m}$ is relevant. Only the top element of this column is non-zero. Hence, we may replace the product by $Z_{m} \zeta_{x}$, in which $Z_{m}$ is the upper left element of $Z_{m}$ and $\zeta_{x}$ is the upper element of $\underline{\xi}_{x}$. Hence, in this particular case,

$u_{\mathrm{riv}}=-g \beta \zeta_{x} \sum_{m=1}^{M} f_{m} Z_{m}$

We assume that the surface slope does not vary across the channel and that Coriolis forcing can be considered negligible. In that case, $\zeta_{x}$ and the $Z_{m}$ are independent of the transverse coordinate $y$ so that Eqs. 81 and 83 can be combined to

$$
\zeta_{x}=-\frac{Q h^{2}}{g \beta \sum_{m=1}^{M} \operatorname{Re}\left\{Z_{m}\right\} F_{m} \int_{\text {transect }} h^{3} \mathrm{~d} y}
$$

in which $F_{m}$ is defined in Eq. 48

With this result, along-channel flow profile can be written as

$\operatorname{Re}\left\{u_{\text {riv }}\right\}=h^{2} Q\left(\int_{\text {transect }} h^{3} \mathrm{~d} y\right)^{-1} \frac{\sum_{m=1}^{M} \operatorname{Re}\left\{Z_{m}\right\} f_{m}}{\sum_{m=1}^{M} \operatorname{Re}\left\{Z_{m}\right\} F_{m}}$

Gravitational circulation can be described in a similar fashion. The effect of an along-channel density gradient $\rho_{x}$ is compensated by an along-channel surface slope such that the net discharge equals zero:

$$
\begin{aligned}
0= & \int_{\text {transect }} h \int_{\sigma_{0}}^{1} \operatorname{Re}\left\{u_{\mathrm{grc}}\right\} \mathrm{d} \sigma \mathrm{d} y \\
u_{\mathrm{grc}}= & -g \beta h \frac{\rho_{x}}{\rho_{0}} \sum_{m=1}^{M} X_{m} f_{m}-g \beta \zeta_{x} \sum_{m=1}^{M} Z_{m} f_{m}
\end{aligned}
$$

with $X_{m}$ the upper left element of matrix $X_{m}$ and in which $u_{\text {grc }}$ is the flow profile corresponding to gravitational circulation. The two expressions in Eq. 86 can be combined to

$\operatorname{Re}\left\{u_{\mathrm{grc}}\right\}=-g \beta h \frac{\rho_{x}}{\rho_{0}}\left(\sum_{m=1}^{M} \operatorname{Re}\left\{X_{m}\right\} f_{m}-\frac{A}{h} s_{M} \sum_{m=1}^{M} \operatorname{Re}\left\{Z_{m}\right\} f_{m}\right)$

in which
$S_{M}=\frac{\sum_{k=1}^{M} \operatorname{Re}\left\{X_{k}\right\} F_{k}}{\sum_{k=1}^{M} \operatorname{Re}\left\{Z_{k}\right\} F_{k}} \quad ; \quad A=\frac{\int_{\text {trassect }} h^{4} \mathrm{~d} y}{\int_{\text {transect }} h^{3} \mathrm{~d} y}$

In the above expression 87 for the flow profile $u_{g r c}$ corresponding to gravitational circulation, the summation containing $X_{m}$ reflects the part driven by a horizontal density gradient. The summation with $Z_{m}$, on the other hand, reflects that part of the flow that is induced by the surface slope.

The total along-channel velocity profile is obtained by superimposing the river discharge Eq. 85 and the gravitational circulation Eq. 87. We may divide this total into a surface slope-induced part $u \zeta$ and a density gradient-induced part $u_{\rho}$ according to

$u_{\zeta}=g \beta h \frac{\rho_{x}}{\rho_{0}} \frac{A}{h} s_{M} \sum_{m=1}^{M} \operatorname{Re}\left\{Z_{m}\right\} f_{m}+h^{2} Q\left(\int_{\text {transect }} h^{3} \mathrm{~d} y\right)^{-1} \frac{\sum_{m=1}^{M} \operatorname{Re}\left\{Z_{m}\right\} f_{m}}{\sum_{m=1}^{M} \operatorname{Re}\left\{Z_{m}\right\} F_{m}}$

$u_{\rho}=-g \beta h \frac{\rho_{x}}{\rho_{0}} \sum_{m=1}^{M} \operatorname{Re}\left\{X_{m}\right\} f_{m}$

Both parts share the property that the magnitude of the velocity increases monotonously with height above the bottom. Both parts reflect uni-directional flow, but that is not necessarily the case for their superposition. As the surface slope and density gradient are counteracting forces, the total flow profile may be either uni- or bi-directional. Which of these forms occurs depends on the relative magnitudes of the two forces involved, the local water depth $h$ and the geometry of the channel's cross section (reflected by $h^{3}$ integrated over the transect and $A$ ). More in particular, if we consider an estuary through which a river discharges into the sea, like Chesapeake Bay, the following three types of flow profiles may occur at individual locations along a transect:

1. A uni-directional landward flow (the density gradientinduced landward flow is stronger than the surface slopeinduced outflow).

2. A bi-directional flow (in the upper part of the water column the surface slope-induced outflow is stronger than the density gradient-driven flow, whereas it is just the other way around in the lower part of the water column).

3. A uni-directional seaward flow (the surface slope-induced outflow is stronger than the density gradient-induced landward flow).

The transition between cases 1 and 2 is characterized by a zero flow velocity at the water surface and for the transition between cases 2 and 3 that is a zero vertical gradient of the 
horizontal flow velocity at the bottom. This follows immediately from the aforementioned property that both $u \zeta$ and $u_{\rho}$ increase monotonously with height above the bottom. These transitions are given by

$$
\operatorname{Re}\left\{u_{\zeta}(1)+u_{\rho}(1)\right\}=0 \quad ; \quad \operatorname{Re}\left\{\left.\frac{\partial}{\partial \sigma}\left(u_{\zeta}+u_{\rho}\right)\right|_{\sigma=\sigma_{0}}\right\}=0
$$

respectively. These relations can be elaborated to

$Q\left(g \frac{\rho_{x}}{\rho_{0}} \int_{\text {transect }} h^{3} \mathrm{~d} y\right)^{-1}=\frac{h}{\bar{v}_{t}}\left(\frac{\sum_{m=1}^{M} \operatorname{Re}\left\{X_{m}\right\} C_{m}}{\sum_{m=1}^{M} \operatorname{Re}\left\{Z_{m}\right\} C_{m}} \sum_{m=1}^{M} \operatorname{Re}\left\{Z_{m}\right\} F_{m}-\frac{A}{h} \sum_{m=1}^{M} \operatorname{Re}\left\{X_{m}\right\} F_{m}\right)$

with $C_{m}=f_{m}(1)$ for the flow velocity at the surface and $C_{m}=$ $\mathrm{d} f_{m} / \mathrm{d} \sigma$ evaluated at $\sigma=\sigma_{0}$ for the velocity gradient at the bottom. All summations over $m$ in the above expressions depend only on the shape functions $f_{m}$ defined in Eq. 41. If we use the shape functions corresponding to a parabolic eddy viscosity distribution, it follows for Eq. 92 that

$$
\begin{aligned}
& Q\left(g \frac{\rho_{x}}{\rho_{0}} \int_{\text {transect }} h^{3} \mathrm{~d} y\right)^{-1}=\frac{h}{\bar{\nu}_{\mathrm{t}}}\left(0.5807-0.6127 \frac{A}{h}\right) ; \\
& Q\left(g \frac{\rho_{x}}{\rho_{0}} \int_{\text {transect }} h^{3} \mathrm{~d} y\right)^{-1}=\frac{h}{\bar{\nu}_{\mathrm{t}}}\left(0.6542-0.6127 \frac{A}{h}\right)
\end{aligned}
$$

for the velocity at the surface and the velocity gradient at the bottom, respectively.

We apply this result to Chesapeake Bay. For the concerned cross-channel profile and with the river discharge and alongchannel density gradient as specified in Table 1, we find that

$$
\begin{aligned}
& \int_{\text {transect }} h^{3} \mathrm{~d} y=1.91 \times 10^{6}\left[\mathrm{~m}^{4}\right] ; \int_{\text {transect }} h^{4} \mathrm{~d} y=2.16 \times 10^{7}\left[\mathrm{~m}^{5}\right] ; \\
& Q\left(g \frac{\rho_{x}}{\rho_{0}} \int_{\text {transect }} h^{3} \mathrm{~d} y\right)^{-1}=120.1\left[\frac{\mathrm{s}}{\mathrm{m}}\right]
\end{aligned}
$$

Hence, the transition between 1 and 2 (from a unidirectional flow, dominated by a density gradient to bidirectional flow) is given by

$120.1\left[\frac{\mathrm{s}}{\mathrm{m}}\right] \bar{v}_{\mathrm{t}}=0.5807 \mathrm{~h}-6.9290[\mathrm{~m}]$

and that for the transition between 2 and 3 (from bidirectional flow to a uni-directional flow dominated by river discharge), it holds that

$120.1\left[\frac{\mathrm{s}}{\mathrm{m}}\right] \bar{v}_{\mathrm{t}}=0.6542 h-6.9290[\mathrm{~m}]$

(with $h$ in metres and $\bar{v}_{\mathrm{t}}$ in square metres per second).

From the above two expressions, it can be seen that the water depths at which the transitions occur depend on the scale of the eddy viscosity. This particular property is used in the present study to tune the AM to observed flow patterns by varying the eddy viscosity scale. As an illustration, it is shown in Fig. 17 in which part of the considered transect of Chesapeake Bay which type of flow profile may be expected, depending of the prescribed eddy viscosity scale.

One of the things the central graph in Fig. 17 and the underlying theory show is that the combination of river discharge, along-channel density gradient, channel geometry and eddy viscosity scale influence the height above the bottom up to which the density gradient-driven landward flow reaches. In all model computations performed for the present study, the eddy viscosity scale is the only one of these four items that has not been assigned a value beforehand. Instead it is used as a calibration parameter. Its value has been tuned to have the model reproduce the observed pattern of uni- and bi-directional tide-residual flow. Evidently, the result of this tuning must be interpreted in relation to the presumed shape of the vertical distribution of eddy viscosity (parabolic in this case).

In the centre of the considered Chesapeake Bay transect, for instance, the landward flow reaches all the way up to the surface if $\bar{v}_{\mathrm{t}}$ is less than about $0.01 \mathrm{~m}^{2} / \mathrm{s}$. This landward flow is suppressed entirely by river discharge for $\bar{v}_{\mathrm{t}}>0.018 \mathrm{~m}^{2} / \mathrm{s}$ approximately. For intermediate values of this scale, the flow in the centre of the channel is bi-directional. Comparing this with the observed tide-residual along-channel flow (Fig. 17) suggests an eddy viscosity scale of about $0.013 \mathrm{~m}^{2} /$ s. However, this figure is based on the IM, not including tidal forcing, Coriolis deflection, advection, horizontal diffusion and transverse density gradient. These mechanisms are taken into account in the AM. As calibration of this AM resulted in a lower value of the eddy viscosity scale $\left(0.006 \mathrm{~m}^{2} / \mathrm{s}\right)$, these mechanisms apparently hamper penetration of the density gradient-driven landward flow up to the water surface.

\section{Appendix 6: Eddy viscosity shape functions}

The shape function for the parabolic profile is written as

$$
\varphi(\sigma)=\varphi_{\mathrm{p}} \sigma\left(\sigma_{\mathrm{s}}-\sigma\right) \quad ; \quad \varphi_{\mathrm{p}}^{-1}=\int_{\sigma_{0}}^{1} \sigma\left(\sigma_{\mathrm{s}}-\sigma\right) \mathrm{d} \sigma
$$




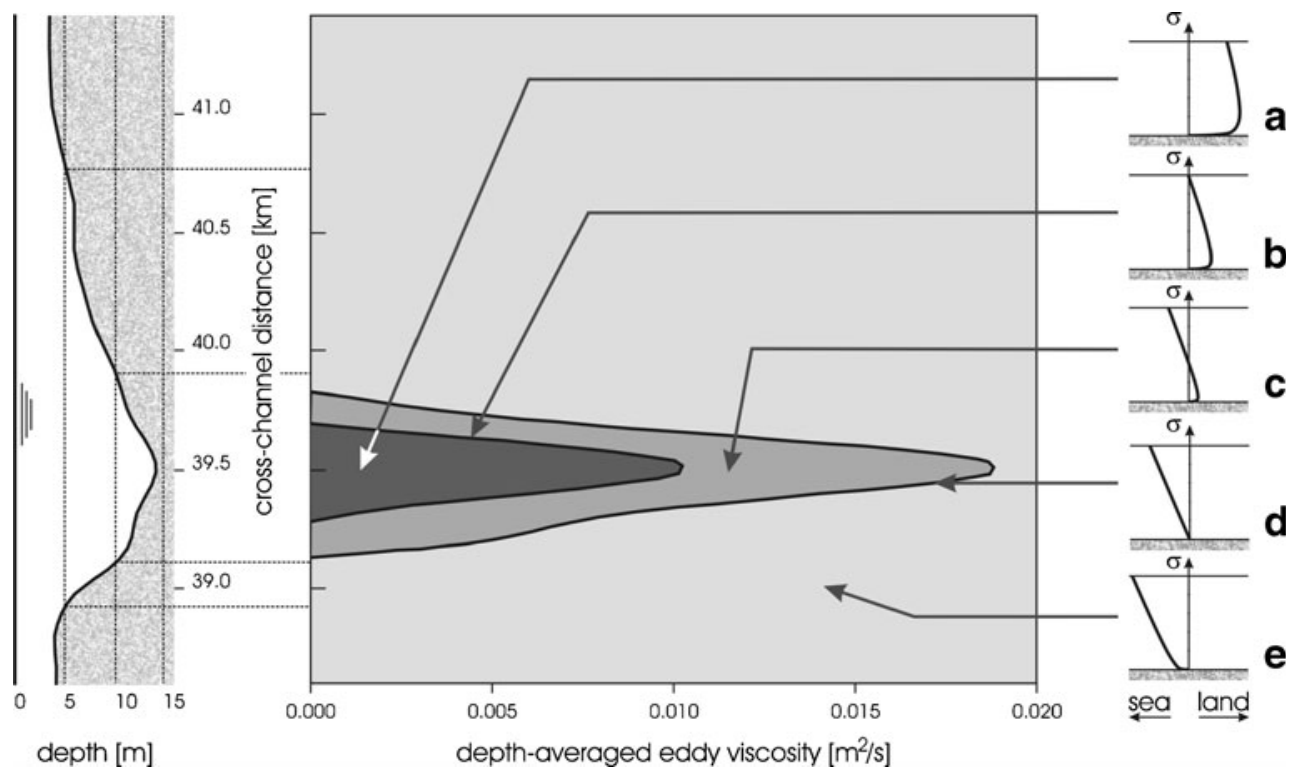

Fig. 17 Application of the IM to the studied transect of Chesapeake Bay (shown on the left) for a steady flow, driven by a combination of an along-channel density gradient and river discharge (explained in "Appendix 5"). Vertical sections taken from the graph in the middle show for the corresponding eddy viscosity scale in which part of the transect which type of flow profile ( $a$ through $e$ on the right-hand side) may be expected. In the dark grey area, a landward, density gradientdriven flow dominates of river outflow (profile $a$ ). The opposite is encountered in the white area (profile $e$ ). In the light grey area, the flow is bi-directional: In the lower part of the water column, the density gradient prevails whereas the river outflow dominates in the upper part (profile $c$ ). Profiles $b$ and $d$ are transitions between these three areas
The coefficient $\varphi_{\mathrm{p}}$ ensures that the integral of the shape function over the range from the level of zero intercept ( $\sigma=\sigma_{0}$, the level at which the bottom boundary condition is applied) to the surface level $(\sigma=1)$ equals 1 . This is consistent with defining $v_{\mathrm{t}}$ as the depth-mean eddy viscosity. One of the zero-crossings of the parabola is chosen just above the water surface level $\left(\sigma_{\mathrm{s}}=1.001\right.$ in all our model computations mentioned in the present paper). A physical argument for choosing $\sigma_{\mathrm{s}}>1$ is that the turbulence scale at the water surface cannot become infinitely small. In addition, it prevents singularities in the model computations.

The other zero crossing is located at $\sigma=0$, which is slightly below the level $\sigma_{0}$ where the no-slip condition is applied. The distance between the two is chosen in correspondence with the bottom roughness. This approach is similar to Prandtl's mixing length concept applied to plane shear flow. This type of flow is given by

$0=-g \frac{\mathrm{d} \zeta}{\mathrm{d} x}+\frac{\bar{v}_{t}}{h^{2}} \frac{d}{\mathrm{~d} \sigma}\left(\varphi \frac{\mathrm{d} u}{\mathrm{~d} \sigma}\right) \quad ; \quad u\left(\sigma_{0}\right)=\left.\frac{\mathrm{d} u}{\mathrm{~d} \sigma}\right|_{\sigma=1}=0$

If we use Eq. 97 for the shape of the eddy viscosity distribution, the velocity profile becomes

$u(\sigma)=-\frac{g h^{2}}{\bar{v}_{\mathrm{t}}} \frac{\mathrm{d} \zeta}{\mathrm{d} x} \int_{\sigma_{0}}^{\sigma} \frac{1-s}{\varphi(s)} \mathrm{d} s$

in which $h$ is the water depth and $x$ is the direction of the surface slope. Elaborating the above expression and taking the limit for $\sigma_{\mathrm{s}} \rightarrow 1$ yields the well-known logarithmic velocity profile.

The value of $\sigma_{0}$ is chosen such that Eq. 99 yields the same depth-averaged velocity as follows from the Chézy relation that applies to the same type of flow:

$\bar{u}|\bar{u}|=C_{\mathrm{h}}^{2} R \frac{\mathrm{d} \zeta}{\mathrm{d} x}$

in which $\bar{u}$ is depth-averaged velocity, $R$ is hydraulic radius (approximately equal to the water depth $h$ if the width of the channel is large compared to its depth) and $C_{\mathrm{h}}$ is the Chézy coefficient. It is related to the bottom roughness length $k_{\mathrm{s}}$ according to

$C_{\mathrm{h}}=\frac{1}{\kappa} \sqrt{g} \ln \left(\frac{12 R}{k_{\mathrm{s}}}\right)$

( $\kappa$ is Von Karman's constant) which is known as the WhiteColebrook formula (see various textbooks on the subject). By combining Eqs. 99, 100 and 101, $\sigma_{0}$ can be determined for a prescribed $k_{\mathrm{s}}$.

The second option we address is a vertically invariant eddy viscosity. This schematisation is encountered in the literature in combination with either a no-slip or a partialslip bottom boundary condition. In our study, we consider both alternatives. For the no-slip case, we use the eddy viscosity profile given in Fig. $2 \mathrm{c}$ and apply the bottom boundary conditions mentioned in the previous section (boiling down to $u_{r}=u_{\theta}=u_{z}=0$ at $\sigma=\sigma_{0}$ ). 
A partial-slip condition is commonly applied at some relative height $\sigma=\sigma_{\mathrm{b}}$ above the bottom. This level divides the water column into an upper layer in which a vertically invariant eddy viscosity is assumed and a lower layer in which the eddy viscosity tends towards zero with decreasing height above the bottom in a way that agrees with Prandtl's mixing length concept (see expression 98 and Fig. 2 b). The velocity profile within this layer hence attains a shape similar to the one given by Eq. 99. This layer is not included in the actual flow computations, but properties of the velocity profile inside it are used to specify the partial-slip condition imposed at the lower end (at $\sigma=\sigma_{\mathrm{b}}$ ) of the upper layer. The eddy viscosity is assumed continuous at this level (see Fig. 2 b) and so are the horizontal velocity and its vertical gradient. Combined with Eq. 99, it then follows that at the transition level $\sigma=\sigma_{\mathrm{b}}$ the horizontal flow velocity component should satisfy

$$
u\left(\sigma_{\mathrm{b}}\right)=\left.c_{\mathrm{b}} \frac{\partial u}{\partial \sigma}\right|_{\sigma=\sigma_{\mathrm{b}}} \quad ; \quad c_{\mathrm{b}}=\int_{\sigma_{0}}^{\sigma_{\mathrm{b}}} \frac{1-s}{\varphi_{*}(s)} \mathrm{d} s\left(\frac{1-\sigma_{\mathrm{b}}}{\phi_{*}\left(\sigma_{\mathrm{b}}\right)}\right)^{-1}
$$

in which $\varphi_{*}$ stands for the combined parabolic and vertically invariant eddy viscosity distribution depicted in Fig. $2 \mathrm{~b}$ and given by

$\varphi_{*}(\sigma)=\left\{\begin{array}{cc}\varphi_{\mathrm{c}} \sigma_{\mathrm{b}}\left(\sigma_{\mathrm{s}}-\sigma_{\mathrm{b}}\right) & ; \quad \sigma_{\mathrm{b}} \leq \sigma \leq 1 \\ \varphi_{\mathrm{c}} \sigma\left(\sigma_{\mathrm{s}}-\sigma\right) & ; \quad \sigma_{0} \leq \sigma<\sigma_{\mathrm{b}}\end{array}\right.$

with

$\varphi_{\mathrm{c}}^{-1}=\frac{1}{1-\sigma_{0}}\left(\sigma_{\mathrm{b}}\left(\sigma_{\mathrm{s}}-\sigma_{\mathrm{b}}\right)\left(1-\sigma_{\mathrm{b}}\right)+\sigma_{\mathrm{b}}^{2}\left(\frac{1}{2} \sigma_{\mathrm{s}}-\frac{1}{3} \sigma_{\mathrm{b}}\right)-\sigma_{0}^{2}\left(\frac{1}{2} \sigma_{\mathrm{s}}-\frac{1}{3} \sigma_{0}\right)\right)$

In common applications of a partial-slip condition, Eq. 102 is imposed at $\sigma=\sigma_{\mathrm{b}}$ and the part of the water column below this level is not considered explicitly in the flow computations. One might say that it is represented by the coefficient $c_{\mathrm{b}}$.

Expression 102 shows that the ratio $c_{\mathrm{b}}$ of the flow velocity and its vertical gradient at the level $\sigma_{\mathrm{b}}$ at which the partial-slip condition is applied depends on the structure of the flow below that level. In other words, the ratio $c_{\mathrm{b}}$ cannot be chosen arbitrarily. Proper flow modelling requires that the value assigned to $c_{\mathrm{b}}$ is consistent with the actual flow profile below $\sigma_{\mathrm{b}}$ or at least a plausible estimate of that profile.

In addition, the level $\sigma=\sigma_{\mathrm{b}}$ at which the partial-slip condition is applied is not impermeable. A condition to be imposed on the flow velocity component normal to this level is therefore not self-evident. In our model computations, we deal with this by considering the entire water column from $\sigma=\sigma_{0}$ up to $\sigma=1$ and prescribe the full eddy viscosity profile (Eq. 103) in combination with demanding that all velocity components are zero at $\sigma=\sigma_{0}$. This way, we simulate partial slip. An additional benefit of this approach is that it allows evaluation of the flow through the level $\sigma=\sigma_{\mathrm{b}}$ which may contribute to finding an appropriate boundary condition for the velocity component normal to this level consistent with partial slip.

\section{References}

Abramowitz M, Stegun I (1970) Handbook of mathematical functions. Dover, New York

Bowden KF (1965) Horizontal mixing in the sea due to a shearing current. J Fluid Mech 21:83-95

Boyd JP (2000) Chebyshev and Fourier spectral methods. Dover, New York

Burchard H, Hetland RD, Schulz E, Schuttelaars HM (2011) Drivers of residual estuarine circulation in tidally energetic estuaries: straight and irrotational channels with parabolic cross-section. J Phys Oceanogr. doi:10.1175/2010JPO4453.1

Chen S-N, Sanford LP (2009) Lateral circulation driven by boundary mixing and the associated transport of sediments in idealized partially mixed estuaries. Cont Shelf Res 29:101-118

Chen S-N, Sanford LP, Ralston DK (2009) Lateral circulation and sediment transport driven by axial winds in an idealized, partially mixed estuary. J Geophys Res 114:C12006

Cheng P, Valle-Levinson A (2009) Influence of lateral advection on residual currents in microtidal estuaries. J Phys Oceanogr. doi:10.1175/2009/JPO4252.1

Cheng P, Wilson RE, Chant RJ, Fugate DC, Flood RD (2009) Modeling influence of stratification on lateral circulation in a stratified estuary. J Phys Oceanogr. doi:10.1175/2009JPO4157.1

Davies AM (1987) On extracting current profiles from vertically integrated numerical models. Coast Eng 11:445-477

Friedrichs CT, Hamrick JM (1996) Effects of channel geometry on cross sectional variation in along-channel velocity in partially mixed tidal estuaries. In: Aubrey DG, Friedrichs CT (eds) Buoyancy effects on coastal and estuarine dynamics. American Geophysical Union, Washington, DC, pp 265281

Fugate DC, Friedrichs CT, Sanford LP (2007) Lateral dynamics and associated transport of sediment in the upper reaches of a partially mixed estuary, Chesapeake Bay, USA. Cont Shelf Res 27:679698

Huijts KMH, Schuttelaars HM, de Swart HE, Valle-Levinson A (2006) Lateral trapping of sediment in tidal estuaries: an idealized model. J Geophys Res. doi:10.1209/2006JC003615

Huijts KMH, Schuttelaars HM, de Swart HE, Friedrichs CT (2009) Analytical study of the transverse distribution of along-channel and transverse residual flows in tidal estuaries. Cont Shelf Res 29:89-100

Huijts KMH, de Swart HE, Schramkowski GP, Schuttelaars HM (2011) Transverse structure of tidal and residual flow and sediment concentration in estuaries. Ocean Dyn. doi:10.1007/s10236011-0414-7

Ianniello JP (1979) Tidally induced residual currents in estuaries of constant breadth and depth. J Mar Res 35:755-786

Lerczak JA, Geyer WR (2004) Modeling the lateral circulation in straight, stratified estuaries. J Phys Oceanogr 34:14101428

Schramkowski GP, Huijts KMH, de Swart HE, Schuttelaars HM (2007) A model comparison of flow and lateral sediment trapping in estuaries. In: Dohmen-Janssen CM, Hulscher SJMH (eds) 
River, coastal and estuarine morphodynamics: RCEM 2007. Taylor \& Francis Group, London, pp 413-420

Schramkowski GP, de Swart HE, Schuttelaars HM (2010) Effect of bottom stress formulation on modelled flow and sediment trapping in crosssections of tide-dominated estuaries. Ocean Dyn 60:205-218

Stelling GS, Van Kester JATM (1994) On the approximation of horizontal gradients in sigma co-ordinates for bathymetry with steep bottom slopes. Int $\mathrm{J}$ Numer Methods Fluids 18:915-935

Valle-Levinson A, Reyres C, Sanay R (2003) Effects of bathymetry, friction and rotation on estuary-ocean exchange. J Phys Oceanogr $33: 2375-2393$

Wong KC (1994) On the nature of transverse variability in a coastal plain estuary. J Geophys Res 99C7:14209-14222 